\title{
Kriminalpolitik und Strafrechtswissenschaft
}

\author{
Michael Kubiciel ${ }^{1}$
}

\section{Große Strafrechtsreform in Einzelschritten}

\section{Verflüssigung des Strafrechts}

Ausbreitung abstrakter Gefährdungsdelikte, akzessorische Straftatbestände, die außerstrafrechtliche Normen, aber keine exakt umrissenen Rechtsgüter schützen, eine Verquickung von Straf- und Polizei- zu einem Kriminalpräventionsrecht: All diese Entwicklungen zeigen, dass das Strafrecht im Wandel ist, dass sich die Ordnung des Strafrechts verändert. ${ }^{2}$ Doch schwindet nicht nur die Ordnung des Strafrechts; auch viele Maximen der Strafrechtswissenschaft verlieren ihre Erklärungskraft, wenn sie auf das sich zunehmend ,verflüssigende“ Strafrecht angewandt werden sollen. ${ }^{3}$

Dieser Prozess der Verflüssigung des Strafrechts hat sich in der Ära der dritten Großen Koalition beschleunigt: Jedenfalls hat der Gesetzgeber keinem anderen Rechtsgebiet in den Jahren 2013 bis 2017 derart große Aufmerksamkeit zuteilwerden lassen wie dem Strafrecht. Mit einer Vielzahl von Einzelschritten hat die Bundesregierung in der 18. Legislaturperiode nichts Geringeres vollzogen als die größte Strafrechtsreform der letzten zwanzig Jahre. ${ }^{4}$ Die Änderungen erstrecken sich von den Sanktionsregeln - Rassismus als Strafzumessungskriterium, Fahrverbot als Nebenstrafe, elektronische Aufenthaltsüberwachung für Terroristen, Recht der Vermögensabschöpfung - bis hin zum Besonderen Teil des StGB. Dort wurden unter anderem das Staatsschutz-, Sexual- und Korruptionsstrafrecht reformiert, die geschäftsmäßige Suizidbeihilfe pönalisiert und die Vorschriften

1 Eine kürzere Fassung ist erschienen in JZ 2018, $171 \mathrm{ff}$.

2 Umfassend dazu Zabel: Die Ordnung des Strafrechts (2017), passim.

$3 \mathrm{Zu}$ den rechtswissenschaftstheoretischen Folgen eines Umfangs mit einem „flüssigen" Gegenstandsbereich Zabel, in: Lohse/Reydon, Grundriss Wissenschaftsphilosophie (2017), S. 167, 170 und passim.

4 Zur 6. Strafrechtsreform des Jahres 1996 Arzt, ZStW Bd. 111 (1999), S. 757 ff.; Bussmann, StV (1999), S. 613 ff.; Freund, ZStW Bd. 109 (1997), S. 455 ff.; Kreß, NJW (1998), S. 633 ff.; Schroeder, NJW (1999), S. 3612 ff. 
gegen Widerstand gegen Vollstreckungsbeamte geändert. Dem Wettbetrug und der Spielmanipulation widmete der Gesetzgeber eigene Straftatbestände, dem Doping ein ganzes Gesetz. Kurz vor Ende der Legislaturperiode wurden das Veranstalten von und die Teilnahme an verbotenen Kraftfahrzeugrennen sowie die Behinderung von Rettungsmaßnahmen kriminalisiert und der Straftatbestand des Wohnungseinbruchsdiebstahls verschärft. ${ }^{5}$ Aus dem Blickwinkel der Rechtswissenschaft betrachtet, stehen die Jahre 2013 bis 2017 folglich für eine Renaissance der Kriminalpolitik.

\section{Triebkräfte der Kriminalpolitik}

Während die Große Strafrechtsreform der 1960er und 1970er Jahre vor allem vom Diskurs um seinerzeit als zukunftsweisend geltende Konzepte (Spezialprävention, Rechtsgüterschutz) bestimmt wurde, ${ }^{6}$ folgen die jüngsten Änderungen des StGB keinem erkennbaren kriminalpolitischen Leitbild. Es waren vielmehr ganz unterschiedliche Gründe, die den Gesetzgeber zum Handeln veranlassten. In manchen Bereichen, vor allem dem Korruptionsstrafrecht, hatte die Große Koalition die längst überfällige Umsetzung internationaler und europäischer Rechtsakte nachzuholen, ${ }^{7}$ in anderen Fällen - Wettbetrug, Autorennen etc. - waren es die von Karl Binding im Jahr 1903 beschriebenen Wellen des täglichen Lebens, die dem Gesetzgeber neue Kriminalitätsphänomene vor die Füße spülten. ${ }^{8}$ In einem Fall - Korruption im Gesundheitswesen - schloss der Gesetzgeber

5 Übersicht über die Reformen des StGB während der dritten Großen Koalition bei Bachmann, RuP (2017), S. 416 ff. Überblick über die Rechtspolitik der Großen Koalition bei Maas, ZRP (2017), S. 130 ff.; ders., RuP (2017), S. 261, 264 ff.

6 Zur Großen Strafrechtsreform zuletzt Greco/Roger, JZ (2016), S. 1125 ff.; Kubiciel, in: Löhnig/Preisner/Schlemmer, Reform und Revolte (2012), S. $217 \mathrm{ff}$.

7 S. zu $§ 299$ StGB Hoven, NStZ (2015), S. 553 ff.; Gaede, NZWiSt (2014), S. 280 ff.; Kubiciel, ZIS (2014), S. 667 ff. Zur Auslandsbestechung Korte und Kubiciel, in: Hoven/Kubiciel, Das Verbot der Auslandsbestechung (2015), S. 45 ff., 63 ff.; zur Mandatsträgerbestechung Kubiciel/Hoven, NK (2014), S. 340 ff.; Sinner, in: FS für Kargl (2015), S. 559 ff.

8 Vgl. Binding: Lehrbuch des gemeinen deutschen Strafrechts Bd. 1 (1902), S. 20. Zum Sportwettbetrug Kubiciel, WiJ (2016), S. 256 ff.; ders., SpuRt (2017), S. 188 ff.; Rübenstahl, JR (2017), S. 264 ff. Zum Phänomen illegaler Autorennen Ceffinato, ZRP (2016), S. 201; Jansen, jurisPR-StrafR (13/2016), Anm. 1; dies., NVZ (2017), S. 214 ff.; Kubiciel, jurisPR-StrafR (16/2016), Anm. 1; Mitsch, DAR (2017), S. 70 ff.; Preuß, NZV (2017), S. 105 ff. 
eine durch eine Entscheidung des Großen Senats des BGH entstandene, europaweit einmalige Strafbarkeitslücke, ${ }^{9}$ in einem anderen - der Nachstellung ( $\$ 238$ StGB) - führte eine äußerst restriktive Anwendungspraxis $\mathrm{zu}$ einem korrigierenden Eingriff in den Tatbestand. ${ }^{10}$ Der umstrittenen Reform des Sexualstrafrechts verhalfen schließlich vermeintliche und echte Skandale sowie die „Kölner Silvesternacht“ zum Durchbruch. ${ }^{11}$ Diese und andere Gesetzesänderungen waren mithin auch eine Antwort auf Verunsicherungen der Gesellschaft ${ }^{12}$ und einer damit einhergehenden Veränderung ihrer „straffundierenden Einstellungen“"13.

\section{Systematische Kriminalpolitik?}

a) Kritik der Wissenschaft

Ein Gesetzgeber, der auf derart unterschiedliche Impulse reagiert, weil er nach den Regeln des internationalen Rechts bzw. den Geboten der politischen Klugheit auf diese reagieren muss, kann nicht systematisch arbeiten. Dessen war sich schon Binding bewusst. ${ }^{14}$ Gleichwohl beklagt die Strafrechtswissenschaft der Gegenwart, die Kriminalpolitik werde stärker von Pragmatik als Prinzipienorientierung bestimmt ${ }^{15}$ und verwende das Strafrecht zunehmend als Mittel einer „wahlkampforientierten Symbolik."16

9 Kubiciel, in: ders./Hoven, Korruption im Gesundheitswesen (2017), S. 69 ff.

10 Dazu Kubiciel/Borutta, KriPoZ (2016), S. 194 ff. Krit. dazu Steinberg, JZ (2017), S. $676 \mathrm{ff}$.

11 Hoven/Weigend, JZ (2017), S. $182 \mathrm{ff}$.

12 Zu diesem Gemütszustand der Nation Maas, AnwBl. (2016), S. 546; Weigend, FS für Frisch (2013), S. 17, 27. S. ferner Großmann: Liberales Strafrecht in komplexen Gesellschaften (2016), S. 40 ff.

13 Dazu Frisch, FS für Jung (2007), S. 189, 210.

14 S. Binding, Fn. 7. Ferner Gärditz: Der Staat Bd. 49 (2010), S. 331, 353. S. auch Kindhäuser, ZStW Bd. 129 (2017), S. 382, 386: Wenn die Strafrechtspolitik das „parteipolitisch artikulierte Bedürfnis“ spiegele, sei die Strafrechtsentwicklung der „legislatorischen Dezision“ anheimgegeben.

15 Hoven, DRiZ (2017), S. 280, 281. S. auch Kindhäuser, ZStW Bd. 129 (2017), S. 382, 385 f.: ,panikartige Attacken der Strafgesetzgebung“, ,zunehmende Beliebigkeit in der Kriminalisierung“. Ähnlich Bachmann, RuP (2017), S. 416, 438.

16 Greco/Roger, JZ (2016), S. 1125, 1127. S. auch Hoven, DRiZ (2017), S. 280, 281: „spontane und aktionistische Symbolpolitik wie das undurchdachte und womöglich verfassungswidrige Sexualstrafrecht". Ähnlich die Rechtspolitikerin der FDP Leutheusser-Schnarrenberger, StV (2017), Editorial Heft 10. 
Fischer vermisst „eine echte Idee vom Recht und von seiner Ausgestaltung, vom Zusammenhang der großen Programme mit den kleinen Schritten (...). “17 Ohne eine solche Idee drohe das StGB zu einem „Flickenteppich ohne System“ zu werden. ${ }^{18}$ Mit diesen ästhetischen Bedenken hat es jedoch nicht sein Bewenden. Vielmehr verlangt der in der Strafe liegende, besondere Eingriff in den Rechtsstatus des Delinquenten auch nach einer besonderen, d.h. freiheitstheoretisch fundierten und axiologisch geschlossenen Rechtfertigung. ${ }^{19}$ Das ,treuherzige Eingeständnis ,Das machen wir zufällig gerade so" "sei keine ausreichende Begründung, so Zaczyk. ${ }^{20}$ Vielmehr habe sich die Gesetzgebung - einer verbreiteten Auffassung zufolge - an einem vorpositiven „Über-Strafrecht“ zu orientieren, das dem Zugriff einer parlamentarischen Mehrheit entzogen sei. ${ }^{21}$

\section{b) Unzureichende Fundierung der Kritik}

Indes sind die Schwierigkeiten, die entstehen, wenn der Gesetzgeber auf die Einhaltung von vorpositiven Leitbildern verpflichtet werden soll, seit langem bekannt. Zunächst muss die Wissenschaft für Vorgaben, die über jene der Verfassung hinausgehen, einen Geltungsgrund nennen können, ${ }^{22}$ wenn sie nicht lediglich unverbindliche Empfehlungen aussprechen will. ${ }^{23}$ Eine anschluss- und tragfähige Erklärung, wie eine (straf)rechtsphilosophische Konzeption gleichsam aus sich selbst heraus und ohne Akt des

17 Fischer, Zeit Online v. 20.9.2017, abrufbar unter: http://www.zeit.de/2017/39/heik o-maas-justizminister-legislaturperiode-wuerdigung (zuletzt abgerufen: 17.10.2017).

18 Heinrich, KriPoZ (2017), S. 4, 9.

19 Pawlik: FS für Jakobs (2007), S. 469 ff.; Zaczyk: Der Staat Bd. 50 (2011), S. 295, 299.

20 Treffend Zaczyk: Der Staat Bd. 50 (2011), S. 295, 299.

$21 \mathrm{Zu}$ diesen Forderungen der Wissenschaft Donini: Jahrbuch Juristische Zeitgeschichte Bd. 3 (2001/2002), S. 408, $411 \mathrm{f}$.

22 Dazu am Beispiel der Straftheorien Gärditz: Der Staat Bd. 49 (2010), S. 331 ff., am Beispiel des Rechtsgutsbegriffs Engländer, ZStW Bd. 127 (2015), S. 616 ff.; Stuckenberg, ZStW Bd. 129 (2017), S. 350, $351 \mathrm{f}$.

23 Für den letztgenannten Weg jüngst Kindhäuser, ZStW Bd. 129 (2017), S. 382, 385 f.; Prittwitz, ZStW Bd. 129 (2017), S. 390, 396 („Appell zur rechtspolitischen Klugheit"). 
Verfassungs- oder Gesetzgebers in den Modus der Rechtsgeltung gelangen kann, ist jedoch nicht ersichtlich. ${ }^{24}$

Aber selbst wenn ein solcher Geltungsgrund gefunden werden könnte, sind die Inhalte, auf die sich die Wissenschaft einigt, häufig von einer stupenden Allgemeinheit. So kann die große Mehrheit der Strafrechtswissenschaftler zwar dem Satz zustimmen, das Strafrecht solle der Freiheit des Einzelnen dienen. ${ }^{25}$ Bei der - notwendigen - kriminalpolitischen Konkretisierung gelangt die Wissenschaft jedoch selten zu einem Konsens. Erfasst beispielsweise der Tatbestand der Nachstellung strafunwürdiges Verhalten und ist daher selbst eine unzulässige Verkürzung der Freiheit von Stalkern ${ }^{26}$ oder schützt er im Gegenteil den Kern aller Freiheitsdelikte das Recht, sein Leben autonom zu gestalten?27 Dienen, um ein anderes Beispiel zu nennen, die $\S \S 216,217$ StGB dem Schutz des Einzelnen vor nicht-vollzugsreifen, d.h. nicht hinreichend autonomen Sterbeverlangen oder sind sie im Gegenteil unverhältnismäßige Eingriffe in die Grundrechte sterbewilliger Personen? ${ }^{28}$ Die Fragen ließen sich fortsetzen. Sie zeigen, dass sich die Freiheitsorientierung des Strafrechts zwar leicht postulieren lässt, dass aber weder die Grenzen der äußeren Freiheitssphären einer Person noch die positiven Voraussetzungen ihrer realen Freiheit feststehen und daher ohne weiteres von der Strafrechtswissenschaft feststellbar sind. Der Gesetzgeber muss den Umfang negativer und positiver Pflichten vielmehr festlegen. Kriminalpolitik ist folglich nicht wesentlich Deduktion, sondern Dezision.

Bei dieser Entscheidung kann sich der Gesetzgeber nicht an dem in der Strafrechtswissenschaft weithin konsensfähigen Satz orientieren, der Einsatz des Kriminalrechts müsse das Mittel der letzten Wahl (ultima ratio)

24 Treffend Stuckenberg, ZStW Bd. 129 (2017), S. 349, 353: Es sei bislang nicht gezeigt worden, ,auf welche Weise der Sprung aus der Welt der Gedanken in die Sphäre der Rechtsgeltung geschehen sollte.".

25 Dass diesem Satz ,fast alle deutschen Strafrechtswissenschaftler“ zustimmen können, konstatiert zutr. Weigend: FS für Frisch, S. 19.

26 Sonnen, in: Nomos Kommentar, StGB, Bd. 2 (2017), § 238 Rn. 27.

27 Kubiciel, jurisPR-StrafR (8/2016); ders./Borutta, KriPoZ (2016), S. 194.

28 Dazu einerseits Kubiciel, ZIS (2016), S. 396 ff.; Engländer: FS für Schünemann (2014), S. 583 ff.; andererseits Hoven, ZIS (2016), S. 1 ff.; Saliger: Selbstbestimmung bis zuletzt? (2015). Vermittelnd Duttge, ZStW Bd. 129 (2017), S. 448, $452 \mathrm{ff}$. 
sein. ${ }^{29}$ Denn die Operationalisierung des Ultima-ratio-Grundsatzes setzt, neben anderem, die Erfüllung einer Aufgabe voraus, an deren Bewältigung die Strafrechtswissenschaft scheitert: eine einigermaßen klare Grenzlinie zu artverwandten Rechtsgebieten, namentlich dem Recht der Ordnungswidrigkeiten, zu ziehen. ${ }^{30}$ So erkennt die Strafrechtswissenschaft zwar an, dass die Strafe begrifflich eine Reaktion auf eine Straftat darstellt und die Tatschuld die Strafvollstreckung legitimiert und auch das Strafmaß bestimmt. ${ }^{31}$ Auf die Frage aber, ob beispielsweise der Verstoß gegen das (bußgeldbewehrte) Kartellverbot des $\S 1$ GWB eine geringere oder qualitativ andere Schuld beinhalte als ein Vergehen nach $\S 298$ StGB, weiß sie keine Antwort zu geben. ${ }^{32}$ Die straftheoretischen Unklarheiten nehmen noch dadurch zu, dass die Strafe einerseits mit Präventionszielen gerechtfertigt wird, andererseits Strafe Schuld voraussetzen soll. Dieses Nebeneinander von Prävention und Retribution lässt keine axiologisch geschlossene Strafbegründung zu, sondern mündet in den Widersprüchen der sog. Vereinigungstheorien. ${ }^{33}$ Damit nicht genug. Wird die Strafe primär präventionstheoretisch begründet, wird das Strafrecht zu einem staatlichen Präventionsinstrument unter anderen: Nicht nur das Strafrecht schützt Rechtsgüter; dies tut, unter anderem, auch das Ordnungswidrigkeitenrecht. Auf einer solch diffusen straftheoretischen Grundlage lässt sich dem Gesetzgeber schwerlich vorhalten, er verwische die Grenzen zwischen strafwürdigem Verhalten und einer bloßen Ordnungswidrigkeit.

Diese offene Flanke vermag die Strafrechtswissenschaft mit ihrem gängigen strafrechtstheoretischen Instrumentarium nicht zu schließen. In die-

29 Umfassend zum Ultima-ratio-Prinzip Kaspar: Verhältnismäßigkeitsgrundsatz und Grundrechtsschutz im Präventionsstrafrecht (2014), S. 243 ff. - Die Geltung dieses Prinzips relativieren (in unterschiedlichem Ausmaß) Jahn/Brodowski, JZ (2016), S. 969, 978 ff.; Kubiciel, ZStW Bd. 129 (2017), S. 473, 487 ff. Aus Sicht des Verfassungsrechts Gärditz, JZ (2016), S. $641 \mathrm{ff}$. , der zudem die geringe bis fehlende praktische Bedeutung in der Rspr. des BVerfG deutlich macht. Dass das Prinzip ,allgemein anerkannt“ ist (so Bachmann, RuP (2017), S. 416, 438), lässt sich daher weder mit Blick auf die Wissenschaft noch mit Blick auf die potenziellen „Anwender" des Prinzips - Gesetzgeber und BVerfG -sagen.

30 Zum Scheitern dieser Versuche Jahn/Brodowski, JZ (2016), S. 969, 972 f.

31 Näher dazu Hörnle: Tatproportionale Strafzumessung (1999); Kindhäuser: Strafrecht AT (2017), § 21 Rn. 1; Kubiciel: Die Wissenschaft vom Besonderen Teil des Strafrechts (2013), S. 247 ff., jeweils mit weiteren Nachweisen. Aus verfassungsrechtlicher Sicht erhellend Wolff, AöR Bd. 123 (1999), S. 55 ff.

32 Zu den Gründen Kubiciel, ZStW Bd. 129 (2017), S. 473, 489.

33 Pawlik, ZIS (2011), S. 262, 263. 
sem war lange der Satz zentral, das Strafrecht dürfe nur Rechtsgüter schützen. ${ }^{34}$ Inzwischen setzt sich mehr und mehr die Einsicht durch, dass der Rechtsgutsbegriff keine Richtschnur für den Gesetzgeber sein könne, da er offen für beliebige Inhalte ist und die verwendeten Konkretisierungselemente (Recht, Interesse, Wert) selbst vage sind. ${ }^{35}$ Es ist bezeichnend, dass diejenigen, die den Rechtsgutsbegriff zuletzt verteidigt haben, in ihm keinen feststehenden Begriff erblicken, sondern einen Platzhalter für kriminalpolitische Strafwürdigkeitsdiskurse. ${ }^{36}$ Als Platzhalter bezeichnet das Rechtsgut lediglich einen Ort, an dem über das Für und Wider einer Kriminalisierung diskutiert wird, aber keinen feststehenden Begriff, der diesen Diskurs anleiten oder gar ersetzen könnte. Als Leitlinie für die Politik ist er demzufolge ungeeignet.

Der Vorwurf der Strafrechtswissenschaft, dem Gesetzgeber fehle eine leitende Idee und kriminalisiere daher, was nicht strafwürdig sei, steht folglich auf einer schwachen Grundlage. Zum einen scheint es so, als folge die Wissenschaft selbst keinem kriminalpolitischen Konzept, sondern ihrer Intuition. Letztere sollte man nicht geringschätzen, denn wenn das $\mathrm{Ob}$ und Wie der Gesetzgebung einen weiten Ermessensspielraum belässt,

34 Dezidiert zuletzt Roxin, JöR Bd. 59 (2011), S. 1 ff.; Schünemann, ZIS (2016), S. 654, 659 ff. Umfassend zur Rechtsgutslehre Jakobs: Rechtsgüterschutz? (2012), S. 7 ff., s. insbes. S. 15 f.; nunmehr auch in: Pawlik: Strafrechtswissenschaftliche Beiträge (2017), S. $123 \mathrm{ff}$.

35 Zur Offenheit Jakobs, S. 15 f., Fn. 33: „chamäleonhafte Wandelbarkeit“. S. ferner Kubiciel, S. 51 ff., Fn. 31; Pawlik: Das Unrecht des Bürgers (2012), S. 130 f.; Stuckenberg, ZStW Bd. 129 (2017), S. 349, 357. Dieses Problem ist - obgleich von der herrschenden Meinung rund vier Jahrzehnte lang verdrängt - seit mehr als 100 Jahren bekannt, s. nur die im Jahr 1886 geprägte Formel vom Rechtsgut als „Proteus“, der bald diese, bald jene Gestalt annehme, bei von Liszt: Strafrechtliche Vorträge und Aufsätze, Bd. 1, (1970), S. 224; fast wortgleich 50 Jahre später Welzel, wiederabgedruckt in: Abhandlungen zum Strafrecht und zur Rechtsphilosophie (1975), S. 135 sowie Amelung: Rechtsgüterschutz und Schutz der Gesellschaft (1972), S. 94 f., 130 ff. - Schünemann, ZIS (2016), S. 654, 662, verweist hingegen auf einen allgemein akzeptierten Begriffskern, der in den Tatbeständen zum Schutz von Leib, Leben und Freiheit zu finden sei, was auf den Gedanken hinausläuft, das Strafrecht habe die personale Freiheit und deren Voraussetzungen zu schützen (vgl. Roxin: Strafrecht AT I (2006), § 2 Rn. 7; zu den Schwierigkeiten bei der Konkretisierung dieses Gedankens s. oben bei Fn. 24).

36 Siehe Kudlich, ZStW Bd. 127 (2015), S. 635, 651; Martins, ZStW Bd. 125 (2013), S. 234, 248; ähnlich Großmann, S. 74, Fn. 11. Siehe bereits Vogel: FS für Roxin (2001), S. 105, 114. Kritik an der Maßstabslosigkeit der diskurstheoretischen Begründung von Rechtsgütern bei Engländer: FS für Neumann (2017), S. 547, 554 ff. 
innerhalb dessen es keine zwingende Lösung gibt, spielt das Einschätzungsvermögen eine erhebliche Rolle. Nur hat die Einschätzung der Wissenschaft keinen größeren legitimationstheoretischen Wert als die Entscheidung des demokratisch legitimierten Gesetzgebers. Letzterer aber hat konkrete, ständig wechselnde soziale Probleme zu lösen und muss sich dabei auch um politische Mehrheiten bemühen. Ihn (ausschließlich oder vorrangig) auf das Modell einer ,neutralen, unpolitischen und beständigen Normsetzung" verpflichten zu wollen, geht daher an der Realität der Gesetzgebung vorbei. ${ }^{37}$

\section{c) Umkehrung der Perspektive}

Die Strafrechtswissenschaft verfügt gegenwärtig über keine idée directri$c e$, mit der sie den Gesetzgeber anleiten und seine „Produkte“ - die Strafgesetze - bewerten könnte. Ihr Leitbegriff (Rechtsgut) ist zu vage und ihr Leitbild „Strafrecht als ultima ratio des Rechtsgüterschutzes“ zu grobschlächtig, um komplizierte Kriminalisierungsfragen zu beantworten. Gegenwärtig verfügt die Strafrechtswissenschaft also nicht über ein Theorierepertoire, mit dessen Hilfe sie die Gesetzgebung anleiten könnte. Angesichts dessen liegt es nahe, vorübergehend die Perspektive zu wechseln: Anstatt von außen, d.h. von einem vorpositiv-wissenschaftlichen Standpunkt auf die Gesetzgebung zu blicken, wird zunächst im neu geschaffenen Recht nach jenen Entwicklungslinien gesucht, die auch in Zukunft die Kriminalpolitik prägen könnten. ${ }^{38}$ Tatsächlich lassen sich solche Tendenzen sichtbar machen.

37 So auch Lepsius, Rg Bd. 25 (2017), S. 152, 153 f.: „Welche Kohärenz, Folgerichtigkeit oder Widerspruchsfreiheit darf man von einem Gesetz erwarten, welches das verfassungsrechtlich vorgesehene Gesetzgebungsverfahren durchlaufen hat?" Die Frage ließe sich weiter zuspitzen, wenn man die internationale oder unionrechtsrechtliche Genese vieler Tatbestände hinzunimmt. Siehe ferner Gärditz, JZ (2016), S 641, $644 \mathrm{f}$.

38 Ähnlicher Ansatz bei Weigend: FS für Frisch, S. 17 ff. 


\section{Entwicklungslinien des Strafrechts}

\section{Expansion des Strafrechts und Kolonialisierung der Lebenswelten}

a) Internationalisierung der Kriminalpolitik

An erster Stelle zu nennen ist die bereits seit längerem zu beobachtende Expansion des Strafrechts. ${ }^{39}$ Sie hat ihren Grund auch in der Internationalisierung der Kriminalpolitik: Für internationale Organisationen und die Europäische Union ist das Strafrecht zuvorderst ein besonders schneidiges Instrument im Werkzeugkasten der Rechtspolitik, dessen Einsatz hauptsächlich davon abhängt, ob die Staaten oder die Verträge (der Europäischen Union) den Einsatz zulassen. ${ }^{40}$ Diese pragmatische Sichtweise führt zur Schaffung neuer und Verschärfung bestehender Straftatbestände.

b) Kompensation für unzureichende gesellschaftliche Selbststeuerung

Doch zeigt sich die Expansion des Strafrechts nicht so sehr in der quantitativen Zunahme von Tatbeständen als vielmehr in dem Umstand, dass das Strafrecht in gesellschaftliche Bereiche vordringt, die zuvor der Selbstregulierung überlassen waren. ${ }^{41}$ Während in den letzten beiden Jahrzehnten Entscheidungen von Unternehmen und Ärzten zunehmend in den Anwendungsbereich des Strafrechts geraten sind, ${ }^{42}$ hat es zuletzt den bislang als „strafrechtsarm“433 geltenden Bereich des Sports erreicht. Die Sportverbände konnten ihre Sphäre so lange im ,handlungskoordinierenden Mechanis-

39 Monographisch Silva-Sánchez: Die Expansion des Strafrechts (2003). S. ferner Kaspar, ZStW Bd. 129 (2017), S. 401: „Zustand einer permanenten StrafrechtsExpansion“; Roxin, in: Neumann/Prittwitz, Kritik und Rechtfertigung des Strafrechts (2005), S. 176 f.; Weigend, StV (10/2016), S. 1 (Editorial): „overcriminalization“. Siehe bereits Peters, ZStW Bd. 77 (1965), $470 \mathrm{ff}$.

40 Kubiciel, NStZ (2007), S. 136 ff.; Meyer, EuR (2011), S. 169 ff.

41 Allg. dazu Raiser: Grundlagen der Rechtssoziologie (2013), S. 368.

42 Eine weitere Expansion des Wirtschaftsstrafrechts prognostiziert Weigend: FS für Frisch, S. $22 \mathrm{ff}$.

43 Treffend Reinhart, in: Fritzweiler/Pfister/Summerer, Praxishandbuch Sportrecht (2014), 8. Teil Rn. 1. 
mus der Verständigung“" ausgestalten, ${ }^{44}$ wie sie selbst die Folgen von Kommerzialisierung und politischer Einflussnahme einzuhegen vermochten. In dem Maße aber, in dem die Verbände die Kontrolle über die Einflüsse von außen verlieren, muss der „Sportpflegestaat ${ }^{\text {“45 }}$ schützend eingreifen - in letzter Konsequenz auch mit dem Mittel des Strafrechts. ${ }^{46}$ Hier, wie auch im Gesundheitswesen, kompensiert das Strafrecht das Versagen gesellschaftlicher Selbstregulierung. Letztere mag - Stichwort: Compliance - funktional notwendig sein, oft stellt sie sich jedoch erst ein, wenn der Staat einen darauf gerichteten (straf-)rechtlichen Impuls aussen$\operatorname{det}^{47}$

\section{c) Gestiegene politische Bedeutung der Sozialmoral}

Ein weiteres aktuelles Beispiel für die Expansion des Kriminalrechts ist das Sexualstrafrecht. In dessen Ausdehnung macht Weigend die Tendenz zur Kriminalisierung „bloß“ moralwidrig-anstößigen Verhaltens aus und erklärt diese Entwicklung mit dem Ziel, unter den Bedingungen einer pluralistischen Gesellschaft Konformität zu fördern. ${ }^{48}$ Auch Kindhäuser meint, dass das Strafrecht zunehmend die schwindenden Normen der Sozialmoral als Mittel zur Lösung sozialer Konflikte zu ersetzen habe, und sieht darin einen Grund für die Ausweitung des Strafrechts. ${ }^{49}$

Indes lässt sich gerade mit Blick auf das Sexualstrafrecht eine Gegenthese aufstellen: Dieser zufolge ist die Ausweitung der Strafbarkeit keine Reaktion auf die Erosion der Sozialmoral, sondern reflektiert im Gegenteil eine gestiegene Sensibilität für sexuelle Übergriffe in einer Gesellschaft, die ihre grundlegenden Verhaltensstandards nicht erst bei einer sexuellen Nötigung (§ 177 StGB a.F.) verletzt sieht, sondern auch bei der (fotografi-

44 Dazu und zu den vom Eindringen zweckrationaler Steuerungsmedien in den „Lebenswelten“" ausgelösten Friktionen Habermas: Theorie des kommunikativen Handelns Bd. 1 (1981), S. 458 f.

45 Treffend Steiner, in: Isensee/Kirchhof; Handbuch des Staatsrechts Bd. 4 (2006), $\S 87$ Rn. 12.

46 Näher dazu Kubiciel, in: Hoven/ders., Korruption im Sport (2018), S. 61 ff.

47 Vgl. zu den Defiziten einer nicht sanktionsbewährten Selbstregulierung SchmittLeonardy: Unternehmenskriminalität ohne Strafrecht? (2013), S. 290 ff.

48 Weigend: FS für Frisch, S. 25.

49 Kindhäuser, ZStW Bd. 129 (2017), S. 382, 385; s. auch Zabel, ZRP (2016), S. 202, 204. 
schen) Wiedergabe einer „teilweise unbekleideten“ jugendlichen Person in „unnatürlicher geschlechtsbetonter Körperhaltung" im Sinne des § 184c Abs. 1 Nr. 1a StGB. Das Strafrecht soll hier also nicht - in der Art eines „Kulturhebels“ - die abschüssige gesellschaftliche Entwicklung ausgleichen, es spiegelt vielmehr zugeschärfte Anforderungen der Sozialmoral. ${ }^{50}$ Dementsprechend lässt sich auch die Ausweitung des Wirtschafts- und Korruptionsstrafrechts nicht als Antwort auf einen angeblich fortschreitenden Sittenverfall im Wirtschaftsleben, sondern vor allem als Reaktion auf eine gewachsene Sensibilität für die Folgen von white collar crimes deuten. ${ }^{51}$ Sollte diese gesellschaftliche Sensibilität weiter zunehmen, könnte unter anderem auch die seit den 1980er Jahren geführte Diskussion wieder aufflammen, ob politische Entscheidungen, die beispielsweise zum Fehlschlag von Bauprojekten führen, in stärkerem Ausmaß als bisher einer strafrechtlichen Haftung zuzuführen sind. ${ }^{52}$

Dass die Bedeutung von Normen der Sozialmoral - von der Wirtschaftsethik bis zur Sexualmoral - weiter steigt, ist angesichts des Veränderungsdrucks, der von außen auf die Gesellschaft einwirkt, wahrscheinlich. Globalisierung, Digitalisierung, Migration und andere, teils disruptiv verlaufende Entwicklungen werden (nicht nur, aber gerade) von der deutschen Gesellschaft als Bedrohung erlebt. ${ }^{53}$ Auf dieses weithin geteilte Gefühl der Bedrohung reagiert eine Gesellschaft Durkheim zufolge, indem sie sich enger zusammenschließt und sich gleichsam um ihre geteilten Werte und Normen versammelt. ${ }^{54}$ Infolgedessen nimmt die politische Bedeutung von Normen der Sozialmoral zu. Es ist daher auch naheliegend, dass Verletzungen solcher Normen zunehmend staatlich sanktioniert werden - auch mit dem Mittel des Strafrechts.

$50 \mathrm{Zu}$ dem durch von Liszt, ZStW Bd. 38 (1917), S. 1, 3, geprägten Bild des Kulturhebels Kubiciel, JZ (2015), S. 64, 68. Zum Verständnis des Strafrechts als „Spiegel“" der Gesellschaft Jescheck: Recht und Staat (1957), S. 4, 8; Welzel: Das deutsche Strafrecht (1954), S. 199.

$51 \mathrm{Zu}$ einem möglichen Grund für die seit den 1990er Jahren gestiegene Sensibilität Kubiciel, ZStW Bd. 129 (2017), S. 473, 491.

52 Kohlmann/Braun: Zur strafrechtlichen Erfassung der Bewirtschaftung öffentlicher Mittel (1979); Schultz, MDR (1979), S. 1981, 372 ff. S. zur Haushaltsuntreue auch Saliger: Parteiengesetz und Strafrecht (2005), S. 218 f.

53 Siehe Fn. 11.

54 Durkheim: Über die Teilung der sozialen Arbeit (1977), S. 144 f.; vgl. ferner Canetti: Masse und Macht, S. $23 \mathrm{ff}$. 
d) Fehlendes Verständnis für die Eigensinnigkeit des Strafrechts

Ein weiterer Grund anzunehmen, dass die Expansion des Strafrechts fortschreiten wird, ist das schwindende Verständnis für die Eigensinnigkeit des Strafrechts in Teilen der Wissenschaft. Befördert wird dieser Verlust nicht nur von den oben geschilderten straftheoretischen Ambivalenzen der sog. Vereinigungstheorie. Dazu kommt ein strafverfassungsrechtliches Begriffsverständnis, dem zufolge unter Strafrecht in Anlehnung an die Kompetenzzuweisung des Art. 74 Abs. 1 Nr. 1 GG ,die Regelung aller, auch nachträglicher, repressiver oder präventiver Reaktionen auf Straftaten“ zu verstehen sein soll. ${ }^{55}$ Aus diesem Blickwinkel betrachtet, verschwindet nicht nur die Grenze zwischen Strafe und Maßregel der Besserung und Sicherung, sondern die besondere Legitimationsbedürftigkeit der Strafvollstreckung schlechthin. Sie, die Strafrechtwissenschaft, tritt dem Gesetzgeber, dem zur Lösung eines sozialen Problems eine breite Auswahl von regulatorischen Möglichkeiten zur Verfügung steht und der das Strafrecht häufig als ein Instrument unter anderen begreift, ${ }^{56}$ mit leeren straftheoretischen Händen gegenüber.

\section{Einzelfallorientierte und sektorspezifische Straftatbestände}

a) Attraktivität

Zugenommen hat in den letzten vier Jahren nicht nur der Umfang des Strafrechts, zugenommen hat auch die Zahl einzelfallorientierter bzw. sektorspezifischer Tatbestände. ${ }^{57}$ So hat der Gesetzgeber die Straflosigkeit der Bestechung und Bestechlichkeit niedergelassener Ärzte nicht durch eine Erweiterung der Vorschriften gegen die Korruption im privaten oder öffentlichen Sektor abgestellt, ${ }^{58}$ sondern auf Heilberufe zugeschnittene Spezialvorschriften geschaffen ( $\S 299 a, 299 b$ StGB). Den von illegalen

55 BVerfGE 109, S. 190, 212. Vgl. dazu Burchard, in: Tiedemann u.a., Die Verfassung des modernen Strafrechts (2016), S. 27, 31; Landau, NStZ (2007), S. 121, 215.

56 Kölbel, in: Tiedemann u.a. (Anm. 54), S. 379, 380, 392.

57 Ob sich schon eine „Überfrachtung“ des StGB mit Spezialtatbeständen feststellen lässt (so Leutheusser-Schnarrenberger, StV (2017), Editorial Heft 10), sei dahingestellt.

58 Dazu Kubiciel, WiJ (2016), S. 1, 8. 
Autorennen ausgehenden Risiken begegnet der Gesetzgeber nicht durch eine Anpassung des Tatbestandes der Straßenverkehrsgefährdung ( $\$ 315 \mathrm{c}$ StGB), sondern mit einer neuen, auf dieses Phänomen zugeschnittenen Vorschrift ( $§ 315 \mathrm{~d}$ StGB) ${ }^{59}$ Auch dem Sportwettbetrug und der korruptiven Wettkampfmanipulation sind eigene Tatbestände gewidmet worden ( $\S 265 \mathrm{c}, 265 \mathrm{~d}$ StGB). ${ }^{60}$

Dieses Vorgehen erklärt sich nicht so sehr dadurch, dass die Umsetzung für den Gesetzgeber „zu schwierig“ oder ,zu zeitraubend“ ist. ${ }^{61}$ Attraktiv ist diese Strategie vor allem deshalb, weil sich für punktuelle Ergänzungen leichter politische Mehrheiten organisieren lassen als für umfassendere Novellierungen. So hätte man der Korruption im Gesundheitswesen auch mit einer Erweiterung des $§ 299$ StGB begegnen können, doch wären davon derart viele Branchen betroffen gewesen, dass der Gesetzgeber diesen Schritt klugerweise gar nicht erst in Erwägung gezogen hat. Zudem lässt sich die Einführung eines Tatbestands, der „punktgenau“ ein besonderes Phänomen zu treffen vorgibt, der Öffentlichkeit leichter vermitteln als die Novellierung einer bestehenden, allgemeiner gefassten Vorschrift.

\section{b) Folgekosten}

Ohne Nebenwirkung ist diese Strategie jedoch nicht. Zunächst führt die Schaffung immer neuer, einzelfallbezogener Straftatbestände zu einer „Aufblähung“ des Strafgesetzbuches ${ }^{62}$ und gesetzesästhetisch unbefriedigenden Wucherungen innerhalb der Legalordnung. In dogmatischer Hinsicht wirft das Nebeneinander von allgemeinen Straftatbeständen und sektorspezifischen bzw. einzelfallorientierten Vorschriften intrikate Konkurrenzprobleme auf. ${ }^{63}$ Schwerer wiegt die kriminalpolitische Sogwirkung,

59 Kubiciel/Hoven, NStZ (2017), S. 439, 445; Mitsch, DAR (2017), S. 70 ff.; Walter, NJW (2017), S. 1350, 1353.

60 Krit. Tsambikakis, in: Hoven/Kubiciel, Korruption im Sport (2018), S. 36 ff.

61 Heinrich, KriPoZ (2017), S. 1, 9.

62 S. das - allerdings schiefe - Bild von der „Aufblähung des Paragrafenwaldes“ bei Steinke, Süddeutsche Zeitung v. 29.6.2017, abrufbar unter: http://www.sueddeutsc he.de/politik/gesetzesverschaerfungen-der-minister-der-unnoetigen-paragrafen-1.3 564394 (zuletzt abgerufen am 27.10.2017). S. bereits Schroeder, NJW (1999), S. 3612, 3614.

63 Zum Verhältnis der $\S \S 299$ a f. StGB zu den $\S \S 299,331$ ff. jüngst Seifert, medstra (2017), S. $280 \mathrm{ff}$. 
die der Gesetzgeber mit der Implementierung von Sondertatbeständen auslöst. ${ }^{64}$ So hat der besondere Schutz, den der Gesetzgeber Vollzugsbeamten und Rettungskräften gegen tätliche Angriffe angedeihen ließ, Forderungen von Verbänden provoziert, auch die Angehörigen ihrer Berufsgruppe besser gegen Übergriffe abzuschirmen.

\section{Reflexive Straftatbestände}

a) Kennzeichen reflexiven Strafrechts

Die dritte Tendenz der Kriminalpolitik zeigt sich an den Wirkungen, welche die Vorschriften gegen die Bestechung im Gesundheitswesen entfaltet haben. Während die Zahl an Ermittlungsverfahren bislang überschaubar ist, hat eine Vielzahl von Ärzten, Kliniken und anderen Marktakteuren ihre Geschäftsbeziehungen und Vertragsausgestaltungen auf ihre Gesetzeskonformität prüfen lassen. Kliniken haben Compliance-ManagementSysteme aufgebaut bzw. diese an das neue regulatorische Umfeld angepasst. Dabei mussten die Normadressaten jene, durchaus erheblichen, Interpretationsspielräume ausfüllen, die das Medizinrecht und die daran akzessorisch anknüpfenden Tatbestände eröffnen. Zivilrechtliche Vereinbarungen und Compliance-Regeln haben die Lücken geschlossen, die das staatliche Recht gelassen hatte. Indem akzessorische Straftatbestände auf diese nicht-staatlichen Regeln Bezug nehmen, sorgen sie einerseits für ein „hardening of soft law" “65; andererseits hat erst das soft law die Straftatbestände hinreichend ausgefüllt und damit ,gehärtet“. Von der seit längerem bekannten und diskutierten Konzeption der regulierten Selbstregulierung unterscheidet sich das hier beschriebene Rechtsphänomen in zweifacher Weise. Zum einen verpflichten die Straftatbestände die privaten Akteure nicht zur Setzung bzw. Umgestaltung eigener Regelungen, sondern initiie-

64 Zum „Kampf“ für eine fortschreitende Ausweitung des Strafrechts durch Vertreter von Partikularinteressen (am Beispiel sog. Moralunternehmer) Silva-Sánchez, S. 28 f., Fn. 38. Krit. gegenüber Einzelfallgesetzen aus demokratietheoretischen Gründen bereits Rousseau: Der Gesellschaftsvertrag (1974), S. 35 f.: Solche Gesetze verlören ihre „natürliche Richtigkeit“.

65 Treffend Schuppert: Governance und Rechtssetzung (2011), S. 6. S. ferner S. Augsberg: Rechtssetzung zwischen Staat und Gesellschaft (2003), S. 230. 
ren diese private Regulierung lediglich. ${ }^{66}$ Diese, den "shadow of the law"67 erhellende Selbstregulierung ist keine Umsetzung einer Rechtspflicht, sondern Wahrnehmung einer Obliegenheit. Zum anderen wirkt die private Regulierung auf die staatliche zurück, indem sie - über das Merkmal ,unlauter" - bei der Auslegung berücksichtigt werden und damit dem Straftatbestand seine vollständige Unrechtsgravur verleihen kann. Diese wechselseitige Beeinflussung ist kennzeichnend für reflexives Recht, das regelgebundene Regulierung um prinzipienorientierte und kooperative Elemente ergänzt. ${ }^{68}$

b) Attraktivität reflexiver Straftatbestände

Obgleich reflexives Recht der gängigen Annahme zuwiderläuft, das Strafrecht stabilisiere mit Hilfe bestimmt formulierter Normen Verhaltenserwartungen, ist mit der Zunahme reflexiver Tatbestände zu rechnen. Denn diese verbinden das Ordnungs- und Rationalitätsversprechen des Governance betreibenden Staates mit dem Wunsch der Bürger und Unternehmen nach Freiraum für eine flexible Umsetzung staatlicher Vorgaben. Sie zeichnen, allgemeiner formuliert, den Wandel vom Subordinationsstaat zum kooperativen Verhandlungsstaat nach. ${ }^{69}$ Zudem reagiert reflexives Recht auf die Krise des regulatorischen Rechts, die sich auch daraus speist, dass sich die schnelle und gelegentlich disruptive Entwicklung von Wirtschaft, Technik und Gesellschaft nicht mit eng gefassten Tatbeständen und starren Normbefehlen kanalisieren lässt. ${ }^{70}$ Schließlich entlastet die staatlich initiierte private Regulierung auch den notorisch überlasteten Apparat staatlicher Rechtsdurchsetzung.

66 Vgl. dazu Baer: Rechtssoziologie (2017), § 6 Rn. 47 (einerseits), Rn. 50 (andererseits).

67 Vgl. Köndgen, AcP Bd. 206 (2006), S. 477, 496; Schuppert, S. 228, Fn. 64.

68 Allgemein dazu Bizer/Führ, in: dies./Hüttig, Responsive Regulierung (2002), S. 1, 4.

69 Zu letzterem Raiser, S. 370, Fn. 40.

70 Zur Steuerungsuntauglichkeit rein interventionistischer Modelle im Wirtschaftsstrafrecht und zu Alternativen Schmitt-Leonardy, S. 286 ff., Fn. 46; Theile: Wirtschaftskriminalität und Strafverfahren (2009), S. 308 ff. 
Schon aus diesen Gründen wird sich diese Entwicklung, die sich an den Rändern des Strafrechts noch deutlicher zeigt als im Kernstrafrecht, ${ }^{71}$ fortsetzen. Hinzu kommt, dass dieses „Aufeinander-Angewiesen-Sein von öffentlicher und privater Regulierung"72 dem Strafrecht nicht fremd ist: Auch „klassische“ Straftatbestände (etwa gegen Leib und Leben) überlassen es dem Bürger, seinen Rechtskreis so zu organisieren, dass aus ihm heraus keine Rechtspositionen Dritter beeinträchtigt werden; sie gründen also letztlich auf dem Prinzip der Selbstkoordination der Gesellschaft. ${ }^{73}$ Gerade im Bereich des Wirtschafsstrafrechts dürfte dieses Prinzip jedoch fortan stärker zur Geltung kommen, da Unternehmen und andere Akteure der Wirtschaftsgesellschaft immer stärker dazu neigen, spezielle, formelle und inhaltlich differenzierte Compliance-Kodizes und anderes soft law zu schaffen. ${ }^{74}$ Es ist funktional vorteilhaft und daher wahrscheinlich, dass Rechtsanwender diese ,sekundären Rechtsquellen“75 verstärkt nutzen werden. Dies wird zugleich den Trend zu reflexiven Straftatbeständen verstärken.

\section{Aufgaben der Strafrechtwissenschaft}

Die Strafrechtsentwicklung verläuft nicht linear. ${ }^{76}$ Daher lässt sich die Kriminalpolitik der Zukunft nicht einfach aus Entwicklungen der Vergangenheit herauslesen. ${ }^{77}$ Dennoch sprechen die oben genannten Gründe dafür, dass sich die Wissenschaft auf weitere Veränderungen des Strafrechts

71 Den $\S 54 \mathrm{a}$ KWG als Vorboten einer prinzipien- statt regelorientierten Gesetzgebung bezeichnend Rönnau, in: Kempf/Lüderssen/Volk u.a., Unbestimmtes Wirtschaftsstrafrecht und gesamtwirtschaftliche Perspektive (2017), S. 51. Zum NetzDG, das soziale Netzwerke unter Bußgeldandrohung zur Einrichtung gesetzlich umrissener Beschwerdemanagement-Systeme verpflichtet, Guggenberg, NJW (2017), S. 2577 ff.; Holznagel, ZUM (2017), S. 615 ff.; Koreng, GRUR-Prax (2017), S. 203 ff.; Kubiciel, jurisPR-StrafR (7/2017).

72 Schuppert, S. 235, Fn. 64.

73 Dazu Pawlik, S. 179 f., Fn. 34.

74 Lüderssen, in: Kempf/Lüderssen/Volk, Gemeinwohl im Wirtschaftsstrafrecht (2013), S. 259, 269 f.

75 Möllers/Hailer, JZ (2012), S. 843, 849.

76 Gärditz: Staat und Strafrechtspflege (2013), S. 30.

77 Näher dazu (am Beispiel der Evolutionstheorie Liszts) Kubiciel, in: Kempf/ Lüderssen/Volk et al., Strafverfolgung in Wirtschaftsstrafsachen (2015), S. 158, $159 \mathrm{ff}$. 
einstellen muss, die keiner systematischen Idee folgen, ${ }^{78}$ sondern entlang der eben skizzierten Entwicklungslinien verlaufen, deren Urheber nicht die Wissenschaft ist. Dies wirft die Frage auf, wie sie sich als Wissenschaft zu den (kleineren und grundlegenderen) Veränderungen des Strafrechts positionieren sollte. Entgegen einer traditionsreichen Meinung kann die Strafrechtswissenschaft nicht scheinbar überzeitlich gültige Ideen, Theorien oder Rechtsbegriffe als Ausgangspunkt ihrer Überlegungen wählen. Wenn sie Aussagen über das Strafrecht und die Kriminalpolitik dieser Gesellschaft treffen will, muss ihre Arbeit vielmehr mit der Betrachtung der Wirklichkeit beginnen.

\section{Betrachten der Wirklichkeit von Gesellschaft, Staat und Politik}

\section{a) Institutionen der Gesellschaft}

Zur gesellschaftlichen Realität gehören Institutionen wie der Wettbewerb, der Kapitalmarkt, das (größtenteils staatlich, aber auch privat organisierte) Gesundheitswesen und der verbands- und vereinsmäßig organisierte Sport. Die Strafrechtswissenschaft kann solche real existierenden, für das Leben der Bürger wichtigen Einrichtungen nicht als normative Anomalien erachten und ihre Schutzwürdigkeit nicht dadurch in Zweifel ziehen, dass sie individuelle Rechte - Leib, Leben, Freiheit, Eigentum - als Paradigmen für zulässige Rechtsgüter ausgibt. Wer ein derart ahistorisches Strafrechtsverständnis als Grundlage des kriminalpolitischen Diskurses wählt, kann nicht zu Aussagen gelangen, die in der Gegenwart anschlussfähig sind. Dass etwa der in Verbänden und Vereinen institutionalisierte Sport, den Millionen Menschen betreiben, der zu den größten Wirtschaftszweigen des Landes gehört und der gerade wegen seiner gesellschaftlichen Bedeutung vom Staat mit Milliarden gefördert wird, nicht schutzwürdig sein soll, ${ }^{79}$ ist eine These, die alle, die es angeht (Sportverbände, -vereine, Rechtspolitiker), nicht nachvollziehen können. Eine Institution von dieser Bedeutung muss der Staat vor existenziellen Bedrohungen schützen dürfen, wenn Verbände und Vereine dazu nicht mehr in der Lage sind und deshalb selbst um Unterstützung bitten. ${ }^{80}$

78 Wie hier Donini, in: Tiedemann et al., S. 87, 89., Fn. 54.

79 In diese Richtung aber Krack, ZIS (2016), S. 540, 543 ff.

80 Näher Kubiciel, KriPoZ (1/2018); a.A. Momsen, KriPoZ (1/2018). 
Die institutionelle Ordnung der Gesellschaft beeinflusst mithin die Gestalt des Strafrechts. ${ }^{81}$ Das gilt auch für das Wirtschaftsstrafrecht: Im modernen Wirtschaftsleben kann das Recht nicht nur die „Ansprüche des einen gegen den anderen" sichern, ${ }^{82}$ sondern muss den Schutzbedürfnissen des Einzelnen und der Wirtschaft durch eine Garantie solcher Normen Rechnung tragen, die für den Bestand von Institutionen (Wettbewerb, Kapitalmarkt etc.) unerlässlich sind. ${ }^{83}$ Die Strafrechtswissenschaft kann die Notwendigkeit institutioneller Garantien nicht ausblenden oder diese für geringwertiger erachten als den Schutz individueller Rechte. Anstatt weiterhin um diese - geschichtlich längst beantwortete - Legitimationsfrage zu kreisen, sollte die Strafrechtswissenschaft den (weniger offensichtlichen) Freiheitsbezug solcher Institutionen nachweisen. Zudem wäre ihr anzuraten, sich mehr Klarheit über die Funktionsbedingungen und -weisen solcher Institutionen zu verschaffen und sich beispielsweise mit den Fragen zu befassen, wie gesellschaftliche Institutionen die strafrechtliche Garantie von Normen intern verarbeiten und welche Rückwirkungen dieser Rezeptionsprozess auf die Anwendung des Strafrechts hat.

b) Kriminalpolitik zwischen internationalen und staatlichen Institutionen

Zur staatlichen Wirklichkeit wiederum gehört die Einsicht, dass die Bundesrepublik in einem dichten Geflecht internationaler Organisationen, Staaten- und Arbeitsgruppen eingebunden ist, das die Kriminalpolitik mitgestaltet und koordiniert. Die Strafgesetzgebung wird daher zunehmend auf internationaler Ebene initiiert und bereits auf der Ebene der Europäischen Union finalisiert. ${ }^{84}$ Die Spielräume des deutschen Gesetzgebers sind dementsprechend klein. Die Betrachtung der Wirklichkeit muss mithin die Beobachtung der Entwicklungen auf der zwischenstaatlichen Ebene sowie die rechtsvergleichende Erfassung internationaler Trends ein-

81 Wilfert: Strafe und Strafgesetzgebung im demokratischen Verfassungsstaat (2017), S. 30, 38 .

82 Treffend der Strafverteidiger und Strafrechtswissenschaftler Alsberg: Wirtschaftsstrafrecht als Problem der Gesetzgebung, Praxis und Wissenschaft (1931), S. 5.

83 Zur Bedeutung dieses Schutzes schon Tiedemann: Verhandlungen des 49. Juristentages Bd. 1 (1972), C 19. Weiterführend Tiedemann, in: ders./et al., S. 13, 18, Fn. 54.

84 Umfassend dazu Meyer: Strafrechtsgenese in internationalen Organisationen (2012). 
schließen. ${ }^{85}$ Überdies muss die Strafrechtswissenschaft die besonderen Finalitäten der einzelnen internationalen Organisationen und die Abstimmungsprozesse zu verstehen lernen, ${ }^{86}$ um die Zielrichtung kriminalpolitischer Aktivitäten zu antizipieren und rechtzeitig, d.h. im Stadium der Beratung von zwischenstaatlichen Übereinkommen oder Aktionsplänen tätig werden zu können. Nur auf diese Weise kann sie die Sichtweise der (deutschen) Strafrechtswissenschaft zu Gehör und gegebenenfalls zur Geltung bringen. Letzteres setzt indes voraus, dass sie ihre Rolle nicht darauf beschränkt, Sonderwege des deutschen Rechts zu verteidigen, sondern ihre Kraft auf die Entwicklung von international anschlussfähigen, rechtsvergleichend informierten Vorschlägen konzentriert. ${ }^{87}$

\section{c) Politische Wirklichkeit}

In den Bereichen, in denen der nationale Gesetzgeber eigene Gestaltungsmacht hat, ist die Art der Regierungskonstellation und das Machtverhältnis zwischen den die Regierung tragenden Parteien von ausschlaggebender Bedeutung für die Kriminalpolitik, wie Nicola Lacey am Beispiel des Vereinigten Königreichs demonstriert hat. ${ }^{88}$ Wenn die Strafrechtswissenschaft auf diese komplexen innerstaatlich-politischen Vorgänge Einfluss nehmen will, muss sie diese Zusammenhänge verstehen und die Nähe zur praktischen Gesetzgebung und Politik nicht scheuen. ${ }^{89}$ Es ist jedenfalls widersprüchlich, dem Gesetzgeber (angebliche) Beratungsresistenz vorzuwerfen ${ }^{90}$ und sich gleichzeitig von der politischen Wirklichkeit abzuschotten.

85 Diese Funktion wäre dem gängigen Katalog von Aufgaben der Rechtsvergleichung hinzuzufügen, vgl. Eser, in: Eser/Perron, Strukturvergleich strafrechtlicher Verantwortlichkeit und Sanktionierung in Europa (2015), S. 929, $970 \mathrm{ff}$.

86 Grundlegend Meyer, S. 56 ff., Fn. 83, sowie am Beispiel zentraler internationaler Akteure S. $91 \mathrm{ff}$.

87 Ambos, GA (2016), S. 177 ff.; Kubiciel, ZStW Bd. 129 (2017), S. 473, 487; Vogel, JZ (2012), S. 25, $28 \mathrm{ff}$.

88 Lacey, Current Legal Problems Bd. 65 (2012), S. 203 ff.

89 Wie hier Ambos, in: Tiedemann et al., S. 321, 327, Fn. 54; Satzger, in: Tiedemann (ebd.), S. 277, 282.

90 S. Zabel, ZRP (2016), S. 202, 203, der auf Hassemer: Strafen im Rechtsstaat (2000), S. 268 ff., verweist. 
2. Reflexion des Selbstverständnisses, methodische Öffnung, Erneuerung des Begriffsapparats

a) Gesetzgebungsskeptizismus oder Annäherung an den Gesetzgeber?

Welche Konsequenzen die Strafrechtswissenschaft aus ihren Beobachtungen zieht, hängt von ihrem Selbstverständnis ab. Wenn sie sich primär als Binnenverwalterin ihres Systems begreift, kann sie dem nationalen und supranationalen Gesetzgeber mit einer unbeugsam kritischen (oder sogar gleichgültigen) Haltung gegenübertreten, weiterhin jede Gesetzesänderung an ihrem System messen und als Fehlleistung des Gesetzgebers zurückweisen. Hält sie hingegen Kritik nicht für ein Mittel der disziplinären Selbstvergewisserung oder einen Selbstzweck und strebt sie gestaltenden Einfluss auf die Kriminalpolitik an, kann sie dem Gesetzgeber nicht mit einem ,genuine disagreement" ${ }^{\star 91}$ begegnen, der jede Verständigung von vornherein ausschließt. Sie muss vielmehr die Weite des politischen Ermessensspielraums sowie den Umfang der Wandelbarkeit des Strafrechts anerkennen und sich bei der Beratung stärker auf das „Wie“, d.h. die konkrete Ausgestaltung der Gesetze konzentrieren, wie es etwa der Arbeitskreis "Alternativ-Entwurf“ seit Jahrzehnten tut, wenn er ausformulierte und begründete Gesetzgebungsvorschläge vorlegt. Letztlich zu entscheiden hat jedoch der Gesetzgeber.

Die Strafrechtswissenschaft hat sich folglich Rechnung darüber abzulegen, ob sie eine primär gesetzgebungskritische oder eine gesetzgebungsberatende Wissenschaft sein will. Beide Optionen haben Vorzüge und Nachteile. Der zuletzt genannte Weg der Annäherung an Gesetzgebung und Politik läuft dem verbreiteten Selbstverständnis der Strafrechtswissenschaft als einer kritischen Disziplin zuwider. ${ }^{92}$ Der erstgenannte Weg hat hingegen den Nachteil, dass ein überzogener Gesetzgebungsskeptizismus den Einfluss der Strafrechtswissenschaft auf die Umgestaltung des Strafrechts von vornherein stark begrenzt, um nicht zu sagen: ausschließt. Da die Gesetzgebung sicher nicht besser wird, wenn die Strafrechtswissenschaft

91 Dazu Dworkin: Law's Empire (1986), S. 45 f.

92 Zum Strafrechtsskeptizismus als (wissenschaftlicher) Lebensform am Beispiel der Frankfurter Schule eindrucksvoll Jahn/Ziemann, JZ (2016), S. 943 ff., insbes. S. 947 mit zahlreichen Nachweisen. Zu den Gründen des gerade in der „Flakhelfer"- und Nachkriegsgeneration verbreiteten Skeptizismus' Pawlik, in: S. Augsberg/Funke, Kölner Juristen im 20. Jahrhundert (2013), S. 225, 240 ff. 
ihres Selbstverständnisses wegen auf jeglichen Einfluss verzichtet, sollte sie einen Wandel durch Annäherung anstreben, anstatt jeden Akt des Gesetzgebers mit einem ,sauren Regen der Kritik“93 zu überschütten. Dauerhafte Fundamentalkritik ist nicht nur wirkungslos, sondern läuft auch Gefahr, zur bloßen Attitüde, zu einem Akt „sterilen Protests“ ${ }^{“ 94}$ zu werden.

b) Verbreiterung des methodischen Instrumentariums

Will sich die Strafrechtswissenschaft im kriminalpolitischen Diskurs Gehör verschaffen, benötigt sie ein theoretisches und methodisches Instrumentarium, das vielschichtiger ist als die in der Vergangenheit bemühten Rechtsguts- und Ultima-ratio-Faustformeln. Die Frage etwa, ob und wie die Korruption im Gesundheitswesen oder das matchfixing im Sport kriminalisiert werden sollen, lässt sich mit Hilfe eines vorpositiven Rechtsgutsbegriffs nicht beantworten.

aa) Sozialwissenschaft und Institutionenökonomie

Von zentraler Bedeutung erscheint zunächst eine stärkere Berücksichtigung von Erkenntnissen der Sozialwissenschaften und der Institutionenökonomie. Gerade die der Strafrechtswissenschaft einst zu großen Hoffnungen Anlass gebenden Sozialwissenschaften sind aus der ,modernen

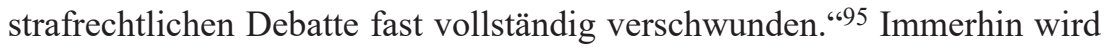
in den letzten Jahren wieder verstärkt die Unterfütterung kriminalpolitischer Vorhaben mit empirischen Erkenntnissen angemahnt bzw. über diese gestritten (s. unten bb.). Mindestens ebenso wichtig wie diese klassische, empirisch-kriminologische Forschung scheint mir jedoch der Zweig der Sozialwissenschaften, der sich mit den Funktionen und Bestandsbedingungen von gesellschaftlichen und staatlichen Institutionen befasst und die Zusammenhänge und Wechselwirkungen verschiedener Institutionen analysiert. So lässt sich über einen Tatbestand wie die Bestechung im Gesundheitswesen nicht gut diskutieren, ohne sich Klarheit über die ideelle, ökonomische und vor allem personale Bedeutung des Gesundheitssystems

93 Arzt, ZStW Bd. 111 (1999), S. 757, 765 sowie 781.

94 Treffend Weigend: FS für Frisch, S. 20.

95 So Roxin, S. 181, Fn. 38. 
zu verschaffen und die Wechselwirkungen mit anderen gesellschaftlichen Institutionen zu betrachten. Diese Institution ist - in sich und wegen ihrer vielfältigen Bezüge $\mathrm{zu}$ anderen Institutionen - nicht nur ausgesprochen komplex, sondern auch für die Freiheit der Einzelnen von großer Bedeutung: Das gilt zum einen für (potenzielle) Patienten, zum anderen aber auch für diejenigen, die ihren Lebensunterhalt mit der Behandlung von Patienten verdienen. Das Gesundheitswesen steht als Institution also ebenso wenig beziehungslos neben einer Person wie die Institutionen Eigentum und Vermögen, sondern stellt den Bürgern Grundbedingungen personaler Freiheitsentfaltung zur Verfügung. Auch die Institution Sport weist einen solchen Freiheitsbezug auf, wenngleich in weniger offensichtlicher Weise als Eigentum und Vermögen.

Anstatt weiterhin über den strafrechtswissenschaftlichen Begriff des Rechtsguts zu räsonieren, könnte die Strafrechtswissenschaft der Kriminalpolitik dadurch dienen, dass sie die tatsächlichen und rechtlichen Besonderheiten, internen Funktionsweisen und rechtliche Ausgestaltung solcher Institutionen analysiert und auf ihren Freiheitsbezug prüft. Diese Funktionsanalyse verspricht mehr Einsichten in die Strafwürdigkeit von (korruptiven oder sonstigen) Angriffen auf Institutionen als die Debatte um einen abstrakten Rechtsgutsbegriff. Überdies legt eine solche Analyse Unterschiede und Gemeinsamkeiten von Institutionen offen und ist damit auch bei der Entscheidung hilfreich, ob einem als strafwürdig erachtetes Phänomen ein eigener Tatbestand gewidmet werden sollte oder ob diesem mit der Ergänzung einer existierenden Vorschrift entgegengetreten werden kann.

\section{bb) Kriminologie}

Wenn einzelne Angriffsformen als potenziell bedrohlich für eine freiheitsermöglichende Institution ausgemacht worden sind, sollte - falls möglich - empirisch überprüft werden, wie weit das sozialschädliche Verhalten verbreitet ist und wie das geltende Recht darauf reagiert. Es sind, anders gewendet, kriminologische Erkenntnisse auszuwerten. Dabei sollte sich die Strafrechtswissenschaft allerdings davor hüten, Fakten zu relativieren oder ganz auszublenden, die ihren Intuitionen oder politischen Überzeugungen zuwiderlaufen. So kann sie die in der Polizeilichen Kriminalstatistik nachweisbare Zunahme tätlicher Angriffe auf Polizeibeamte nicht mit der unbewiesenen Vermutung relativieren, Polizeibeamte neigten zu einer 
übertriebenen Anzeigeerstattung, so dass es die Angriffe vielleicht gar nicht gegeben habe. ${ }^{96}$ Auch die Kritik an der Weite des strafrechtlichen Schutzes individueller Rechte sollte empirische Erkenntnisse berücksichtigen anstatt sie zu übergehen: So kann bei der Diskussion um die $\S \S 216$, 217 StGB nicht unter den Tisch fallen, dass empirischen Untersuchungen zufolge die übergroße Mehrzahl von Sterbeverlangen in Situationen geäußert werden, die gegen und nicht für die Autonomie des Sterbewunsches sprechen. ${ }^{97}$ Und bevor die Debatte um eine Neugestaltung des Verbandssanktionenrechts mit Verweis auf die $\S \S 30,130 \mathrm{OWiG}$ für beendet erklärt wird, sollte man die lückenhafte - um nicht zu sagen: zufällige - Anwendungspraxis des geltenden Rechts analysieren. ${ }^{98}$

\section{cc) Rechtsgrundlagenvergleich}

Größere Bedeutung als bislang muss auch der strafrechtliche Grundlagenvergleich haben. Gemeint ist damit nicht so sehr ein Vergleich der den ausländischen Gesetzen eingeschriebenen Dogmatik, sondern ein Vergleich von Rechtsprinzipien und Maximen der Kriminalpolitik. Ein solcher Vergleich stellt nicht nur den eigenen Theoriebestand in Frage und hält zu dessen ständiger Erneuerung an. ${ }^{99}$ Er kann auch die Voraussage künftiger Entwicklungen erleichtern sowie Grund - und Grenzen! - gemeinsamer (internationaler oder zwischenstaatlicher) Initiativen sichtbar machen. ${ }^{100}$

96 Den Anstieg der polizeilich registrierten Widerstandshandlungen und Köperverletzungen relativieren u.a. Putzke/Rienhoff, JZ (2017), S. 924, 925 f.; Zöller, KriPoZ (2017), S. 143, 144. Gegenkritik dazu bei Kubiciel, jurisPR-StrafR (5/2017).

97 Duttge, ZStW Bd. 129 (2017), S. 448, 453.

98 Dazu demnächst Ergebnisse einer empirischen Untersuchung einer Forschungsgruppe um Henssler/Hoven/Verf./Weigend; s. auch bereits Kubiciel, in: OECD, Consultation on liability of legal persons (2016), S. $81 \mathrm{ff}$. Näher Kubiciel/Hoven, jurisPR-StrafR (23/2017).

99 Hörnle, in: Tiedemann et al., S. 289, 304, Fn. 54.

$100 \mathrm{Zu}$ diesem Grundlagenvergleich Kubiciel, JZ (2015), S. 64, 69 ff. Für eine Aufwertung der Rechtsvergleichung auch Beck/Burchard,/Fateh-Moghadam, in: dies.: Strafrechtsvergleichung als Problem und Lösung (2011), S. 5; Satzger, S. 277 ff., Fn. 88; Vogel, JZ (2012), S. 25, 30. 
dd) Politikwissenschaft

Schließlich darf die Strafrechtswissenschaft nicht ausblenden, dass das Strafrecht, mit Hillgruber gesprochen, ein Aggregatzustand des Politischen ist, ${ }^{101}$ selbst wenn man die Auffassung teilt, dass die Strafe ihre Legitimation ,nicht aus bloßem politischen Kompromiss beziehen kann.“102 Während die Strafrechtswissenschaft sich seit jeher um die Konzeptualisierung eines legitimationstheoretischen Begründungszusammenhanges bemüht und zu diesem Zweck auf die Rechts- und Moralphilosophie zurückgreift, ist ihr die Politikwissenschaft bislang fremd geblieben. ${ }^{103} \mathrm{Da}-$ bei zeigt etwa die Forschung der bereits erwähnten Nicola Lacey die Fruchtbarkeit dieses Wissensgebiets. Man erhält dort Einsichten in den Zusammenhang von politischen Systemen bzw. Koalitionsregierungen einerseits und Kriminalpolitik andererseits und begreift auch, weshalb manche Regierungen die ihnen vor die Füße gespülten Kriminalphänomene aufgreifen und in die Strafrechtspolitik einspeisen, während andere Regierungen an diesen Erscheinungen vorübergehen.

\section{c) Erneuerung des Begriffsapparats}

Schließlich sollte die Strafrechtswissenschaft ihre gegenwärtige Situation zum Anlass nehmen, ihren Begriffsapparat zu erneuern. Insbesondere ist über einen Ersatz für den in vielerlei Hinsicht unglücklichen Begriff „Rechtsgut" nachzudenken. Die Strafe reagiert nämlich nicht auf die Verletzung eines Rechtsguts, verstanden als ein Gut oder Gegenstand, sondern auf die Verletzung eines subjektiven Rechts (als Recht) bzw. einer Norm, die für den Bestand einer Institution wesentlich ist. Damit wird deutlich, dass es im Strafrecht nicht um die generelle Schutzwürdigkeit von Gütern geht, sondern um die Garantie bestimmter subjektiver Rechte und solcher Normen, die für die Stabilität freiheitsermöglichender Institutionen wichtig sind. ${ }^{104}$ Aus diesem Blickwinkel lässt sich die Frage nach der Straf-

101 Hillgruber, VVDStRL Bd. 67 (2008), S. 7, 8.

102 Greco/Roger, JZ (2016), S. 1125, 1133, Zaczyk, Der Staat Bd. 50 (2011), S. 295, $296 \mathrm{f}$.

103 Das Fehlen einer „Kriminalpolitikwissenschaft“ konstatiert mit Recht Prittwitz, ZStW Bd. 129 (2017), S. 390, 399.

104 Näher Kubiciel, S. 168 ff., Fn. 76. 
würdigkeit eines Verhaltens exakter formulieren und beantworten. Zudem zeigt sich, dass das Strafrecht ein primär reaktives Recht ist: Es leistet seinen Beitrag zur Erhaltung der Bedingungen von Freiheit durch eine Stabilisierung existierender Verhaltenserwartungen, es ist hingegen kein Mittel zur Lenkung der Gesellschaft in die Richtung politisch erwünschter sozialer Endzustände. Ein retributives Strafverständnis hat nicht nur den Vorteil axiologischer Geschlossenheit, sondern läuft - kriminalpolitisch gesehen auch einer übermäßigen politischen Aktivierung des Strafrechts zuwider.

\section{d) Alternativen zum Strafrecht suchen!}

Schließlich sollte sich die Strafrechtswissenschaft auch an jener Aufgabe versuchen, die Roxin im Jahr 2000 beiläufig mit dem Begriff der „Subsidiariätswissenschaft" ${ }^{105}$ beschrieben hat: gemeinsam mit Vertretern anderer Teildisziplinen des Rechts sowie der Rechtspraxis nach Alternativen zum Strafrecht zu suchen.

Sich dieser Aufgabe zu widmen ist heute dringlicher denn je. Auf der einen Seite wird das Strafrecht immer weiter ausgebaut: Der Gesetzgeber nutzt es als Impuls- und Steuerungsmittel dort, wo die gesellschaftliche Selbstregulierung versagt (zuletzt: Gesundheitswesen, Sport) und das Ordnungswidrigkeitenrecht wegen seiner geringeren Symbolkraft und - vor allem - seiner geringeren Wirkkraft (Opportunitätsprinzip, Bußgelder) als unzureichend erscheint. Auch bei der Bekämpfung des internationalen Terrorismus greift der Gesetzgeber nicht ohne Not zum Strafrecht, sondern deshalb, weil polizeirechtliche Alternativen an rechtliche und tatsächliche Grenzen stoßen. Die Ausweitung des Strafrechts ist also auch eine Reaktion darauf, dass die neuartigen und zunehmend facettenreicheren Regulierungsprobleme mit den klassischen Alternativen zum Strafrecht (Ordnungswidrigkeiten- und Polizeirecht) nicht angemessen gelöst werden können. Auf der anderen Seite führt die Ausweitung des Strafrechts zu einer Überspannung bzw. Überlastung des Strafrechtssystems: In normativer Hinsicht überspannt die skizzierte Flexibilisierung des Strafrechts das Gesetzlichkeits- und Schuldprinzip und andere das Strafrecht prägende Maximen und führt zu einer „Strapazierung der Begriffe“. In tatsächlicher Hinsicht überlastet die Ausweitung des Strafrechts das Kriminaljustizsys- 
tem, das diesem Druck mit einer Einstellungspraxis - gerade im Bereich der Bagatellkriminalität - abhilft, die bereichsweise die Grenze zu einer De-facto-Entkriminalisierung erreicht. Aus diesen Gründen ist heute mehr denn je - über eine Erweiterung des existierenden Rechtsformenkanons nachzudenken und eine Alternative zum Straf- und Ordnungswidrigkeitenrecht zu entwickeln. Diese Alternative müsste die Schwächen des OWiG ausgleichen, zugleich aber Distanz zum Strafrecht wahren, indem (spezial-)präventiven Zwecken stärkeres Gewicht beigemessen wird als im primär retributiven, schuldbezogenen Strafrecht. Verfahren auf dieser „,neuen“ Spur des Rechts würden also selten in einer Sanktionierung enden, sondern mit einer diversionellen Erledigung und damit sowohl dem Beschuldigten dienen als auch das Strafsystem in tatsächlicher und normativer Hinsicht entlasten. ${ }^{106}$

An der Sanktionierung von Unternehmen und anderen Verbänden lässt sich besonders deutlich machen, dass die existierenden Rechtsspuren nicht ausreichen und eine solche neue Spur Abhilfe schaffen kann. Zum einen ist das Ordnungswidrigkeitenrecht erkennbar nicht auf die Bewältigung unternehmensbezogener Straftaten zugeschnitten: Nicht nur ist das Sanktionensystem zu starr (und wird daher im GWB und anderen Gesetzen immer häufiger und immer deutlicher modifiziert); auch das Verfahrensrecht des OWiG ist auf die Bewältigung massenhaft anfallender Bagatellfälle zugeschnitten, nicht aber auf die Sanktionierung von Unternehmen. Zum anderen aber scheint eine echte Kriminalisierung nicht zweckmäßig, da in Bezug auf Unternehmen das spezialpräventive Ziel der Verbesserung von Unternehmensstrukturen dem strafrechtlichen Schuldausgleich aus mehreren Gründen vorzuziehen ist. Die Lösung liegt folglich in der Schaffung eines Gesetzes, dessen Regeln auf einer Ebene zwischen Straf- und Ordnungswidrigkeitenrecht angesiedelt sind. Der Kölner Entwurf eines Verbandssanktionengesetzes setzt diesen Gedanken um. ${ }^{107}$ Der Versuch, diese Idee $\mathrm{zu}$ verallgemeinern und in einem Gesetzentwurf münden zu lassen, ist aus hiesiger Sicht und nach den Erfahrungen bei der Erarbeitung des Kölner Entwurfes aussichtsreich.

106 Der hier zur Diskussion gestellte Vorschlag deckt sich - partiell - mit der vor rund 20 Jahren von Hassemer (ZRP (1992), S. 378 ff.) skizzierten Idee eines „Interventionsrechts", die allerdings nicht näher konkretisiert worden ist und bislang auch nicht zu konkreten Gesetzesvorschlägen geführt hat. S. zum „Kölner Entwurf eines Verbandssanktionengesetzes“" aber sogleich.

107 Siehe Henssler/Hoven/Kubiciel/Weigend, NZWiSt (2018), Heft 1. 
IV. Fazit

Auch im Bereich des Strafrechts ist die „Gesetzgebung ,old style' nach dem Muster (unpolitischer, beständiger) Kodifikationen“ zu großen Teilen von einer Kriminalpolitik zurückgedrängt worden, die ad hoc auf das Entstehen neuer sozialer Probleme, Veränderungen gesellschaftlicher Bewusstseinszustände und die Eröffnung politischer Chancen reagiert. ${ }^{108} \mathrm{Da}-$ von legen viele Strafrechtsänderungen der vergangenen Jahre beredtes Zeugnis ab. Jedoch sagt der Rückblick auf die Kriminalpolitik der Großen Koalition ebenso viel über den Zustand der Gesellschaft und die Reaktionen der Politik aus wie über die Lage der Strafrechtswissenschaft. Diese hat auf die grundlegenden Veränderungen mit seit Jahrzehnten eingeübten Reflexen und unter Verwendung seit Jahrzehnten (erfolglos) bemühter Formeln reagiert. Von einer Debatte über eine „Neue Strafrechtswissenschaft", wie sie die Verwaltungsrechtslehre vor dem Hintergrund vergleichbar großer Umwälzungen seit eineinhalb Jahrzehnten führt, ${ }^{109}$ ist sie dennoch weit entfernt. Dabei ist die Strafrechtswissenschaft auf die Herausforderungen der zu beobachtenden Umgestaltung des Strafrechts weder konzeptionell noch methodisch ausreichend vorbereitet. Will sie in Berlin und Brüssel (wieder) gehört werden, muss sie ihr Selbstverständnis und ihre Arbeitsweisen überdenken. Tut sie dies, könnte der Renaissance der Kriminalpolitik eine Wiedergeburt der Wissenschaft als Rat- und Impulsgeber folgen.

\section{Literatur}

Alsberg, Max: Wirtschaftsstrafrecht als Problem der Gesetzgebung, Praxis und Wissenschaft, Berlin 1931.

Ambos, Kai, Zur Zukunft der deutschen Strafrechtswissenschaft: Offenheit und diskursive Methodik statt selbstbewusster Provinzialität, GA 2016, S. 177-194.

108 Lepsius, Rg Bd. 25 (2017), S. 153, 156.

109 Zur Neuen Verwaltungsrechtswissenschaft umfassend Voßkuhle, in: HoffmannRiem/Schmidt-Aßmann/Voßkuhle, Grundlagen des Verwaltungsrechts, Bd. 1 (2006), (2012), § 1. Kritische Bestandsaufnahme bei Zimmermann, JZ (2010), S. 1170 ff. S. ferner Lepsius, JZ (2005), S. 1 ff. 
Zur Zukunft der deutschen Strafrechtswissenschaft: Offenheit und diskursive Methodik statt selbstbewusster Provinzialität, in: Tiedemann, Klaus/Schünemann, Bernd/ Rönnau, Thomas (Hrsg.): Die Verfassung moderner Strafrechtspflege, Baden-Baden 2016, S. 321-350.

Amelung, Knut: Rechtsgüterschutz und Schutz der Gesellschaft, Frankfurt am Main 1972.

Arzt, Gunther: Wissenschaftsbedarf nach dem 6. StRG, ZStW 111 (1999), S. 757-785.

Augsberg, Steffen: Rechtssetzung zwischen Staat und Gesellschaft, Berlin 2003.

Bachmann, Mario: Reformen des Strafgesetzbuches durch die dritte „Große Koalition“ - Eine kritische Bilanz, RuP 2017, S. 416-439.

Baer, Susanne: Rechtssoziologie, 3. Aufl., Baden-Baden 2017.

Beck, Susanne/Burchard, Christoph/Fateh-Moghadam, Bijan: Vorwort, in: Beck, Susanne/Burchard, Christoph/Fateh-Moghadam, Bijan (Hrsg.): Strafrechtsvergleichung als Problem und Lösung, Baden-Baden 2011, S. 5-10.

Binding, Karl: Lehrbuch des gemeinen deutschen Strafrechts, 1, 2. Aufl., Leipzig 1902.

Bizer, Kilian/Führ, Martin: Responsive Regulierung - Anforderungen an die interdisziplinäre Gesetzesfolgenforschung, in: Bizer, Kilian/Führ, Martin/Hüttig, Christoph (Hrsg.): Responsive Regulierung, Tübingen 2002, S. 1-21.

Burchard, Christoph: Strafverfassungsrecht - Vorüberlegungen zu einem Schlüsselbegriff, in: Tiedemann, Klaus/Schünemann, Bernd/Rönnau, Thomas (Hrsg.): Die Verfassung moderner Strafrechtspflege, Baden-Baden 2016, S. 27-62.

Bussmann, Kai D., Konservative Anmerkungen zur Ausweitung des Strafrechts nach dem Sechsten Strafrechtsreformgesetz, StV 1999, S. 613-622.

Canetti, Elias: Masse und Macht, München 1994.

Ceffinato, Tobias: Ausdehnung des Verkehrsstrafrechts auf illegale Kraftfahrzeugrennen, ZRP 2016, S. 201-202.

Donini, Massimo: Demokratische und wissenschaftliche Methode einer Verbindung von Strafrecht und Politik, Jahrbuch Juristische Zeitgeschichte 3 (2001/2002), S. 408-437.

Techniken und regulative Modelle eines verfassungsorientierten Strafrechts, in: Tiedemann, Klaus/Schünemann, Bernd/Rönnau, Thomas (Hrsg.): Die Verfassung moderner Strafrechtspflege, Baden-Baden 2016, S. 87-96.

Durkheim, Émile: Über die Teilung der sozialen Arbeit, Frankfurt am Main 1977.

Duttge, Gunnar: Die "geschäftsmäßige Suizidassistenz" (§ 217 StGB): Paradebeispiel für illegitimen Paternalismus!, ZStW 129 (2017), S. 448-467.

Dworkin, Ronald: Law's Empire, Cambridge 1986.

Engländer, Armin: Strafbarkeit der Suizidbeteiligung, in: Hefendehl, Roland/Hörnle, Tatjana/Greco, Luís (Hrsg.): Festschrift für Schünemann, Berlin 2014, S. 583-596.

Revitalisierung der materiellen Rechtsgutslehre durch das Verfassungsrecht?, ZStW 127 (2015), S. 616-635. 
Personale Rechtsgutslehre und normativer Individualismus, in: Saliger, Frank/YoungWhan, Kim/Shing-I, Liu/Mylonopolus, Christos/Keiichi, Yamanaka/Yongliu, Zheng/Tavares, Juarez/Isfen, Osman (Hrsg.): Festschrift für Neumann, Heidelberg 2017, S. 547-560.

Eser, Albin: Teil 4 - Strafrechtsvergleichung: Entwicklung - Ziele - Methoden, in: Eser, Albin/Perron, Walter (Hrsg.): Strukturvergleich strafrechtlicher Verantwortlichkeit und Sanktionierung in Europa, Berlin 2015, S. 929-1136.

Freund, Georg: Der Entwurf eines 6. Gesetzes zur Reform des Strafrechts, ZStW 109 (1997), S. 455-490.

Frisch, Wolfgang: Gesellschaftlicher Wandel als formende Kraft und als Herausforderung des Strafrechts, in: Müller-Dietz, Heinz/Müller, Egon/Kunz, Karl-Ludwig/ Radtke, Henning/Britz, Guido/Momsen, Carsten (Hrsg.): Festschrift für Jung, Baden-Baden 2007, S. 189-214.

Gaede, Karsten: Die Zukunft der europäisierten Wirtschaftskorruption gemäß $§ 299$ StGB - Eine Evaluation des Referentenentwurfs des BMJV vom 13.6.2014, NZWiSt 2014, S. 281-291.

Gärditz, Klaus Ferdinand: Strafbegründung und Demokratieprinzip, Der Staat 2010, S. 331-367.

Demokratizität des Strafrechts und Ultima Ratio-Grundsatz, JZ 2016, S. 641-650.

Staat und Strafrechtspflege, Paderborn 2013.

Greco, Luís/Roger, Benjamin: Strafrechtsreform als Wissenschaft - Zum 50-jährigen Jubiläum des Alternativ-Entwurfs eines Strafgesetzbuches, JZ 2016, S. 1125-1133.

Großmann, Sven: Liberales Strafrecht in komplexen Gesellschaften, Baden-Baden 2016.

Guggenberger, Nikolas: Das Netzwerkdurchsetzungsgesetz in Anwendung, NJW 2017, S. 2577-2582.

Habermas, Jürgen: Theorie des kommunikativen Handelns, Bd. 1, Berlin 1981.

Hassemer, Winfried: Strafen im Rechtsstaat, Baden-Baden 2000.

Kennzeichen und Krisen des modernen Strafrechts, ZRP 1992, S. 378-383.

Heinrich, Bernd: Zum heutigen Zustand der Kriminalpolitik in Deutschland, KriPoZ 2017, S. 4-20.

Henssler, Martin/Hoven, Elisa/Kubiciel, Michael/Weigend, Thomas: Kölner Entwurf eines Verbandssanktionsgesetzes, NZWiSt 2018, S. 1-11.

Hillgruber, Christian: Verfassungsrecht zwischen normativem Anspruch und politischer Wirklichkeit, VVDStRL 2008, S. 7-128.

Hörnle, Tatjana: Tatproportionale Strafzumessung, Berlin 1999.

Plädoyer für eine transnationale Strafrechtswissenschaft, in: Tiedemann, Klaus/Schünemann, Bernd/Rönnau, Thomas (Hrsg.): Die Verfassung moderner Strafrechtspflege, Baden-Baden 2016, S. 289-306.

Hoven, Elisa: Aktuelle rechtspolitische Entwicklungen im Korruptionsstrafrecht - Bemerkungen zu den neuen Strafvorschriften über Mandatsträgerbestechung und $\mathrm{Be}$ stechung im geschäftlichen Verkehr, NStZ 2015, S. 553-561.

Für eine freie Entscheidung über den eigenen Tod, ZIS 2016, S. 1-9. 
Entbehrliche Straftatbestände, DRiZ 2017, S. 280-286.

Hoven, Elisa/Weigend, Thomas: ,Nein heißt Nein“ - und viele Fragen offen, JZ 2017, S. 182-191.

Jahn, Matthias/Brodowski, Dominik: Krise und Neuaufbau eines strafverfassungsrechtlichen Ultima Ratio-Prinzips, JZ 2016, S. 969-980.

Jahn, Matthias/Ziemann, Sascha: Die Frankfurter Schule des Strafrechts: Versuch einer Zwischenbilanz, JZ 2016, S. 943-947.

Jakobs, Günther: Rechtsgüterschutz? Zur Legitimation des Strafrechts, Paderborn 2012.

Jansen, Scarlett: Der Gesetzentwurf zur Strafbarkeit bei nicht genehmigten Autorennen - eine systematische Betrachtung, NZV 2017, S. 214-220.

Jescheck, Hans-Heinrich: Das Menschenbild unserer Zeit und die Strafrechtsreform, Tübingen 1957.

Kaspar, Johannes: Verhältnismäßigkeitsgrundsatz und Grundrechtsschutz im Präventionsstrafrecht, Baden-Baden 2014.

Redundante Tatbestände, ZStW 2017, S. 401-415.

Kindhäuser, Urs: Strafrecht Allgemeiner Teil, 8. Aufl., Baden-Baden 2017.

Straf-Recht und ultima-ratio-Prinzip, ZStW 2017, S. 382-390.

Kölbel, Ralf: Zu Herausforderungen für das regulatorische Strafrecht, in: Tiedemann, Klaus/Schünemann, Bernd/Rönnau, Thomas (Hrsg.): Die Verfassung moderner Strafrechtspflege, Baden-Baden 2016, S. 379-400.

Köndgen, Johannes: Privatisierung des Rechts, AcP 2006, S. 477-525.

Kohlmann, Günther/Braun Johann: Zur strafrechtlichen Erfassung der Bewirtschaftung öffentlicher Mittel, Wiesbaden 1979.

Korte, Matthias: Straftatbestände gegen internationale Bestechung, in: Hoven, Elisa/ Kubiciel, Michael (Hrsg.): Das Verbot der Auslandsbestechung, Baden-Baden 2015, S. 63-76.

Kreß, Claus: Das sechste Gesetz zur Reform des Strafrechts, NJW 1998, S. 633-644.

Kubiciel, Michael: Die Wissenschaft vom Besonderen Teil des Strafrechts, Frankfurt am Main 2013.

Grund und Grenzen strafrechtlicher Anweisungskompetenz der Europäischen Gemeinschaft, NStZ 2007, S. 136-142.

Vergeltung, Sittenbildung oder Resozialisierung? - Die straftheoretische Diskussion um die Große Strafrechtsreform, in: Löhnig, Martin/Preisner, Mareike/Schlemmer, Thomas (Hrsg.): Reform und Revolte, Tübingen 2012, S. 217-230.

Bestechung und Bestechlichkeit im geschäftlichen Verkehr, ZIS 2014, S. 667-673.

Auslandbestechung durch Institutionenschutz: Schutz inländischer Ordnungssysteme durch $\S 355 \mathrm{a}$ StGB, in: Hoven, Elisa/Kubiciel, Michael (Hrsg.): Das Verbot der Auslandsbestechung, Baden-Baden 2015, S. 45-59.

Einheitliches europäisches Strafrecht und vergleichende Darstellung seiner Grundlagen, JZ 2015, S. 64-70. 
Freiheit, Institutionen, abstrakte Gefährdungsdelikte: Ein neuer Prototyp des Wirtschaftsstrafrechts?, in: Kempf, Eberhardt/Lüderssen, Klaus/Volk, Klaus (Hrsg.): Strafverfolgung in Wirtschaftsstrafsachen, Berlin 2015, S. 158-171.

Verschärfung des Anti-Stalking-Tatbestandes, jurisPR-StrafR 8/2016 Anm. 1.

jurisPR-StrafR 16/2016 Anm. 1.

Die Straftatbestände gegen Korruption im Gesundheitswesen: verfassungskonform, kriminalpolitisch angemessen und effektiv?, WiJ 2016, S. 1-11.

Zur Verfassungskonformität des § 217 StGB, ZIS 2016, S. 396-403.

Kubiciel, Michael/Borutta, Nadine: Strafgrund und Ausgestaltung des Tatbestandes der Nachstellung (§238 StGB), KriPoZ 2016, S. 194-198.

OECD (Hrsg.): Consultation on liability of legal persons, Köln 2016.

Unentbehrliches Wirtschaftsstrafrecht, entbehrliche Tatbestände, ZStW 129 (2017), S. 473-492.

Rechtliche Fragen der Korruption im Gesundheitswesen aus Sicht des ärztlichen Berufsrechts, in: Kubiciel, Michael/Hoven, Elisa (Hrsg.), Korruption im Gesundheitswesen, Baden-Baden 2017, S. 69-88.

Der Regierungsentwurf zur Neufassung der $\S \S 113,114$ StGB, jurisPR-StrafR 5/2017 Anm. 1.

Neuartige Sanktionen für Soziale Netzwerke, jurisPR-StrafR 7/2017 Anm. 1.

Kubiciel, Michael/Hoven, Elisa: Gründe für eine Reform des Verbandssanktionenrechts, jurisPR-StrafR 23/2017.

Kubiciel, Michael/Hoven, Elisa: Die Strafbarkeit illegaler Straßenrennen mit Todesfolge, NStZ 2017, S. 439-446.

Neue Haftungsrisiken für Vereine: Die Straftatbestände gegen Sportwettenbetrug und Spielmanipulation, SpuRt 2017, S. 188-194.

Integrität des Sports - Konkretisierung eines Begriffs, KriPoZ 2018, S. 29-31.

Die Straftatbestände des Sportwettenbetrugs und der Manipulation berufssportlicher Wettbewerbe. Legitimation, Interpretation und Folgen, in: Hoven, Elisa/Kubiciel, Michael (Hrsg.): Korruption im Sport, Baden-Baden 2018, S. 61-86.

Kudlich, Hans: Die Relevanz der Rechtsgutstheorie im modernen Verfassungsstaat, ZStW 127 (2015), S. 635-654.

Krack, Ralf: Sportwettenbetrug und Manipulation von berufssportlichen Wettbewerben, ZIS 2016, S. 540-551.

Lacey, Nicola: Political Systems and Criminal Justice: The Prisoners' Dilemma After the Coalition, Current Legal Problems 2012, S. 203-239.

Landau, Herbert: Die Pflicht des Staates zum Erhalt einer funktionstüchtigen Strafrechtspflege, NStZ 2007, S. 121-129.

Lepsius, Oliver: Normpluralismus als Ausdruck der Funktionsrationalität des Rechts, $\operatorname{Rg} 2017$, S. 152-161.

Sozialwissenschaften im Verfassungsrecht - Amerika als Vorbild?, JZ 2005, S. 1-13.

Leutheusser-Schnarrenberger, Sabine. Editorial Heft 10, StV 2017.

von Liszt, Franz: Strafrechtliche Vorträge und Aufsätze, 1, Berlin 1905. 
Einheitliches mitteleuropäisches Strafrecht, ZStW 38 (1917), S. 1-21.

Lüderssen, Klaus: Grenzen der Kriminalisierung gemeinwohlriskanten unternehmerischen Handelns im Rahmen regulierter Selbstregulierungen, in: Kempf, Eberhard/ Lüderssen, Klaus/Volk, Klaus (Hrsg.): Gemeinwohl im Wirtschaftsstrafrecht, Berlin 2013, S. 259-278.

Maas, Heiko: Ein rationales Strafrecht als Ziel der Rechtspolitik bewahren, AnwBl. 2016, S. 546-547.

Modernes Recht für eine moderne Gesellschaft, ZRP 2017, S. 130-134.

Martins, Antonio: Der Begriff des Interesses und der demokratische Inhalt der personalen Rechtsgutlehre, ZStW 125 (2013), S. 234-299.

Meyer, Frank: Das Strafrecht im Raum der Freiheit, der Sicherheit und des Rechts, EuR 2011, S. 169-196.

Strafrechtsgenese in internationalen Organisationen, Baden-Baden 2012.

Mitsch, Wolfgang: Die Strafbarkeit illegaler Rennen de lege lata et ferenda, DAR 2017, S. 70-74.

Möllers, Thomas M. J./Hailer, Sabrina: Möglichkeiten und Grenzen staatlicher und halbstaatlicher Eingriffe in die Unternehmensführung, JZ 2012, S. 841-851.

Momsen, Carsten: Integrität des Sports - Was sollen neue Tatbestände schützen?, KriPoZ 2018, S. 21-28.

Pawlik, Michael: Das Unrecht des Bürgers, Tübingen 2012.

Strafrechtswissenschaftliche Beiträge, Tübingen 2017.

Buchrezension zu: Luís Greco: Lebendiges und Totes in Feuerbachs Straftheorie, ZIS 2011, S. 262-263.

Ulrich Klug (1913-1993): Skeptische Rechtsphilosophie und liberales Strafrecht, in: Augsberg, Steffen/Funke, Andreas (Hrsg.), Kölner Juristen im 20. Jahrhundert, Tübingen 2013, S. 225-242.

Peters, Karl: Beschränkung der Tatbestände im Besonderen Teil, ZStW 77 (1965), S. 470-506.

Prittwitz, Cornelius: Das Strafrecht: Ultima ratio, propria ratio oder schlicht strafrechtliche Prohibition?, ZStW 129 (2017), S. 390-401.

Puschke, Jens/Rienhoff, Jannik: Zum strafrechtlichen Schutz von Polizeibeamtinnen und -beamten, JZ 2017, S. 924-932.

Raiser, Thomas: Grundlagen der Rechtssoziologie, 6. Aufl., Tübingen 2013.

Reinhart, Michael: 8. Teil Sport und Strafrecht, in: Fritzweiler, Jochen/Pfister, Bernhardt/Summerer, Thomas (Hrsg.): Praxishandbuch Sportrecht, 3. Aufl., München 2014.

Rönnau, Thomas: Die politische Wirtschaftsstraftat - gibt es sie?, in: Kempf, Eberhard/ Lüderssen, Klaus/Volk, Klaus/Jahn, Matthias/Prittwitz, Cornelius/Schmidt, Reinhardt (Hrsg.): Unbestimmtes Wirtschaftsstrafrecht und gesamtwirtschaftliche Perspektive, Berlin, Boston 2017, S. 21-33.

Rousseau, Jean-Jacques: Der Gesellschaftsvertrag, Ditzingen 1974.

Roxin, Claus: Schlussbericht, in: Neumann, Ulfrid/Prittwitz, Cornelius (Hrsg.): Kritik und Rechtfertigung des Strafrechts, Berlin 2005, S. 176-193. 
60 Jahre Grundgesetz aus Sicht des Strafrechts, JöR 2011, S. 1-28.

Strafrecht Allgemeiner Teil, 1, 4. Aufl, München 2006.

Rübenstahl, Markus: $\S \S 265$ c und 265d StGB - (Para)Korruptionsstrafrecht zur Bekämpfung des Sportwettenbetrugs und der Manipulation von Berufssportwettbewerben, JR 2017, S. 264-279.

Saliger, Frank: Selbstbestimmung bis zuletzt?, Norderstedt 2015.

Parteiengesetz und Strafrecht, Tübingen 2005.

Satzger, Helmut: Die Rolle einer modernen deutschen Strafrechtswissenschaft im Europäischen und internationalen Kontext, in: Tiedemann, Klaus/Schünemann, Bernd/Rönnau, Thomas (Hrsg.): Die Verfassung moderner Strafrechtspflege, Baden-Baden 2016, S. 277-284.

Schmitt-Leonardy, Charlotte: Unternehmenskriminalität ohne Strafrecht?, Heidelberg, München, Landsberg u.a. 2013.

Schroeder, Friedrich-Christian: Das neue Bild des Strafgesetzbuchs, NJW 1999, S. 3612-3614.

Schünemann, Bernd: Über Strafrecht im demokratischen Rechtsstaat, das unverzichtbare Rationalitätsniveau seiner Dogmatik und die vorgeblich progressive Rückschrittspropaganda, ZIS 2016, S. 654-671.

Schultz, Günther: Kurzbeiträge Strafprozeß-Novelle und gesetzlicher Richter, MDR 1979, S. 371-372.

Schuppert, Gunnar Folke: Governance und Rechtssetzung, 1. Aufl., Baden-Baden 2011.

Seifert, Laura: Das Konkurrenzverhältnis zwischen $\S \S 299 a, 299 b$ StGB und $\S \S 299$, 331 ff. StGB, medstra 2017, S. 280-283.

Silva-Sánchez, Jesús-María: Die Expansion des Strafrechts, Frankfurt am Main 2003.

Sinner, Stefan: Die (un)endliche Geschichte der Abgeordnetenbestechung, in: Albrecht, Peter-Alexis: Festschrift für Walter Kargl, Berlin 2015, S. 559-572.

Sonnen, Bernd-Rüdiger: $\S 238$, in: Kindhäuser, Urs/Neumann, Ulfried/Paeffgen, HansUlrich (Hrsg.): Nomos Kommentar (NK) StGB, 2, 5. Aufl., Baden-Baden 2017.

Steinberg, Georg: Die missratene Änderung des $§ 238$ StGB, JZ 2017, S. 676-680.

Steinke, Ronen: Der Minister der unnötigen Paragrafen, Süddeutsche Zeitung v. 29.6.2017, abrufbar unter: http://www.sueddeutsche.de/politik/gesetzesverschaerf ungen-der-minister-der-unnoetigen-paragrafen-1.3564394.

Steiner, Udo: $\S 87$ Sport und Freizeit, in: Isensee/Kirchhof (Hrsg.), Handbuch des Staatsrechts, 4, 3. Aufl., Heidelberg 2006, S. 727-746.

Stuckenberg, Carl-Friedrich: Rechtsgüterschutz als Grundvoraussetzung von Strafbarkeit?, ZStW 129 (2017), S. 349-363.

Tiedemann, Klaus: Die Verfassung moderner Strafrechtspflege - eine Hinführung, in: Tiedemann, Klaus/Schünemann, Bernd/Rönnau, Thomas (Hrsg.): Die Verfassung moderner Strafrechtspflege, Baden-Baden 2016, S. 13-26.

Welche strafrechtlichen Mittel empfehlen sich für eine wirksame Bekämpfung der Wirtschaftskriminalität?, Ständige Deputation des deutschen Juristentages (Hrsg.): Verhandlungen des 49. Juristentages, 1, 1972, S. C19-C106. 
Theile, Hans: Wirtschaftskriminalität und Strafverfahren, Tübingen 2009.

Tsambikakis, Michael: Überflüssiges Strafrecht, in: Hoven, Elisa/Kubiciel, Michael (Hrsg.): Korruption im Sport, Baden-Baden 2018, S. 37-60.

Vogel, Joachim: Strafgesetzgebung und Strafrechtswissenschaft - Überlegungen zu einer diskurstheoretischen Strafgesetzgebungslehre, in: Roxin, Claus/Schünemann, Bernd (Hrsg.): Festschrift für Roxin, Berlin 2001, S. 105-118.

Strafrecht und Strafrechtswissenschaft im internationalen und europäischen Rechtsraum, JZ 2012, S. 25-31.

Voßkuhle, Andreas: Neue Verwaltungsrechtswissenschaft, in: Hoffmann-Riem, Wolfgang/Schmidt-Aßmann, Eberhard/Voßkuhle, Andreas (Hrsg.), Grundlagen des Verwaltungsrechts, 1, München 2006, S. 1-62.

Walter, Tonio: Der vermeintliche Tötungsvorsatz von "Rasern", NJW 2017, S. 1350-1353.

Weigend, Thomas: Heft 10, (Editorial): „overcriminalization“, StV 2016.

Wohin bewegt sich das Strafrecht? Probleme und Entwicklungstendenzen im 21. Jahrhundert, in: Freund, Georg/Murmann, Uwe/Bloy, René/Perron, Walter (Hrsg.): Festschrift für Frisch, Berlin 2013, S. 17-31.

Welzel, Hans: Abhandlungen zum Strafrecht und zur Rechtsphilosophie, Berlin 1975.

Wolff, Heinrich: Der Grundsatz "nulla poena sine culpa" als Verfassungsrechtssatz, AöR 1999, S. 55-86.

Zabel, Benno: Die Ordnung des Strafrechts, Tübingen 2017.

Strafgesetzgebung und Strafgerechtigkeit, ZRP 2016, S. 202-205.

Philosophie der Rechtswissenschaft, in: Lohse, Simone/Reydon, Thomas (Hrsg.): Grundriss Wissenschaftsphilosophie, Hamburg 2017, S. 167-200.

Zaczyk, Rainer: Demokratieprinzip und Strafbegründung, Der Staat 2011, S. 295-301.

Zöller, Mark A.: Schutz von Vollstreckungsbeamten und Rettungskräften durch das Strafrecht? - Überlegungen zum 52. Gesetz zur Änderung des Strafgesetzbuchs, KriPoZ 2017, S. 143-150. 